\title{
Signature laminar distributions of pathology in frontotemporal lobar degeneration
}

\author{
Daniel T. Ohm ${ }^{1,2}$ - Katheryn A. Q. Cousins ${ }^{2} \cdot$ Sharon X. Xie ${ }^{3} \cdot$ Claire Peterson $^{1,2} \cdot$ Corey T. McMillan $^{2}$. \\ Lauren Massimo ${ }^{2} \cdot$ Katya Raskovsky $^{2}$. David A. Wolk ${ }^{4,5} \cdot$ Vivianna M. Van Deerlin ${ }^{6} \cdot$ Lauren Elman $^{7}$. \\ Meredith Spindler ${ }^{8} \cdot$ Andres Deik $^{8} \cdot$ John Q. Trojanowski ${ }^{4,6,9} \cdot$ Edward B. Lee $^{4,6,9} \cdot$ Murray Grossman $^{2}$. \\ David J. Irwin ${ }^{1,2}$ (1)
}

Received: 11 October 2021 / Revised: 11 December 2021 / Accepted: 28 December 2021 / Published online: 8 January 2022

(c) The Author(s) 2022

\begin{abstract}
Frontotemporal lobar degeneration (FTLD) with either tau (FTLD-tau) or TDP-43 (FTLD-TDP) inclusions are distinct proteinopathies that frequently cause similar frontotemporal dementia (FTD) clinical syndromes. FTD syndromes often display macroscopic signatures of neurodegeneration at the level of regions and networks, but it is unclear if subregional laminar pathology display patterns unique to proteinopathy or clinical syndrome. We hypothesized that FTLD-tau and FTLD-TDP accumulate pathology in relatively distinct cortical layers independent of clinical syndrome, with greater involvement of lower layers in FTLD-tau. The current study examined 170 patients with either FTLD-tau $(n=73)$ or FTLD-TDP $(n=97)$ spanning dementia and motor phenotypes in the FTD spectrum. We digitally measured the percent area occupied by tau and TDP-43 pathology in upper layers (I-III), lower layers (IV-VI), and juxtacortical white matter (WM) from isocortical regions in both hemispheres where available. Linear mixed-effects models compared ratios of upper to lower layer pathology between FTLD groups and investigated relationships with regions, WM pathology, and global cognitive impairment while adjusting for demographics. We found lower ratios of layer pathology in FTLD-tau and higher ratios of layer pathology in FTLD-TDP, reflecting lower layer-predominant tau pathology and upper layer-predominant TDP-43 pathology, respectively $(p<0.001)$. FTLD-tau displayed lower ratios of layer pathology related to greater WM tau pathology $(p=0.002)$ and to earlier involved/severe pathology regions $(p=0.007)$. In contrast, FTLD-TDP displayed higher ratios of layer pathology not related to either WM pathology or regional severity. Greater cognitive impairment was associated with higher ratios of layer pathology in FTLD-tau $(p=0.018)$, but was not related to ratios of layer pathology in FTLD-TDP. Lower layer-predominant tau pathology and upper layer-predominant TDP-43 pathology are proteinopathy-specific, regardless of clinical syndromes or regional networks that define these syndromes. Thus, patterns of laminar change may provide a useful anatomical framework for investigating how degeneration of select cells and corresponding laminar circuits influence large-scale networks and clinical symptomology in FTLD.
\end{abstract}

Keywords Frontotemporal lobar degeneration $\cdot$ Tau $\cdot$ TDP-43 $\cdot$ Laminar pathology $\cdot$ Supragranular $\cdot$ Infragranular

\section{Introduction}

The two most common types of proteinopathies in frontotemporal lobar degeneration (FTLD) are characterized by tau (FTLD-tau) and transactive response DNA-binding protein of $43 \mathrm{kDa}$ (TDP-43) (FTLD-TDP) pathology [22, $57,58]$. FTLD proteinopathies are associated with a diverse

David J. Irwin

dirwin@pennmedicine.upenn.edu

Extended author information available on the last page of the article spectrum of clinical presentations that include dementia phenotypes (i.e., behavioral variant FTD [bvFTD], primary progressive aphasia [PPA]) and motor phenotypes (i.e., amyotrophic lateral sclerosis [ALS], corticobasal syndrome [CBS], progressive supranuclear palsy syndrome [PSPS]) [42]. Accurate antemortem diagnosis of underlying neuropathology still remains a major challenge [59, 67], especially in bvFTD which has an equal likelihood of being caused by FTLD-tau or FTLD-TDP. MRI studies find that FTD syndromes caused by disparate pathologies often share patterns of cortical atrophy and aberrant functional connectivity 
in defined cognitive networks [76, 80, 81, 92, 104]. Since FTLD-type tau or TDP-43 pathology are not yet reliably detected in living patients, it remains unclear how dissimilar proteinopathies cause similar clinical phenotypes. However, detailed postmortem studies of human brains may help elucidate the microanatomy of overlapping clinical heterogeneity in FTLD and guide biomarker development.

Histopathologic studies can test predictions made by models of neural network degeneration that investigate how FTLD-tau and FTLD-TDP patients may present with similar clinical syndromes $[1,52,77,88,101,104]$. One hypothesis to explain clinical similarity in FTLD is that tau and TDP43 pathologic aggregates are anatomically concordant and disrupt the same networks. This implies shared anatomical vulnerability at a microscopic level (e.g., cortical neurons), mesoscopic level (e.g., neural circuits), or macroscopic level (e.g., cortical regions). Indeed, histopathologic studies of bvFTD have shown that select cell populations may contribute to salience network dysfunction [47, 74, 89] after accruing either tau [55, 90] or TDP-43 pathology [68]. An alternative hypothesis is that the same clinically relevant networks are disrupted by different types of neurons, circuits, or regions [21, 88, 101, 104]. In support of this latter hypothesis, recent investigations by our lab and others have found different regional patterns of peak tau and TDP-43 histopathologic burden within the language network in PPA $[29,27,48,49]$ and frontotemporal networks in bvFTD [43, 45]. FTLD proteinopathies may also display different laminar distributions of pathology in the same clinical syndrome and network, but comparative examinations of tau and TDP43 pathology in cortical laminae are lacking.

Laminar cytoarchitecture influences whole-brain network connectivity [35] through diverse neural circuits (e.g., excitatory vs. inhibitory) that originate from select upper layers (I-III) and lower layers (IV-VI) [2, 13-15, 33, 32, 84]. Additionally, heterogeneous isocortical regions with less differentiation (fewer cortical layers) or more differentiation (more cortical layers) likely affect structural connectivity, functional activity, and potentially spread of FTLD pathology [13-15, 32, 26, 35, 36]. Therefore, cortical layers represent an anatomical paradigm for identifying new patterns of histopathologic accumulation within cells and neural circuits poorly understood in the FTLD spectrum. Primary tauopathies (i.e., Pick's disease [PiD], corticobasal degeneration $[\mathrm{CBD}]$, and progressive supranuclear palsy [PSP]) produce widespread tau pathology in most cortical layers, but lower layers often accumulate more Pick cells in PiD, ballooned neurons in CBD, and globose tangles in motor cortex of PSP [4- 7, 34, 37, 38, 97, 98]. In contrast, most TDP-43 proteinopathies accumulate TDP-43 inclusions in upper layers with varied involvement of lower layers [8, $9,22,53,56-58,70,71]$. While these studies suggest that select inclusions accumulate according to cortical layers and provide criteria for subtype classifications, many were limited by their focus to relatively small groups of patients that did not encompass the full pathologic, genetic, and clinical heterogeneity associated with FTLD. As a result, the potential influence of demographics, clinical features, and regions on total pathologic burden across cortical layers in the FTLD spectrum remain poorly understood. Given clinical similarities between FTLD-tau and FTLD-TDP patients, comparative histopathologic studies of FTLD-tau and FTLD-TDP offer a unique clinical-anatomical-pathological framework for understanding mechanisms by which common networks degenerate due to separate proteinopathies.

In this study, we examined the full clinicopathologic spectrum of FTLD to test the hypothesis that severe lower layer pathology is a distinguishing feature of FTLD-tau compared to FTLD-TDP. We calculated ratios of cortical layer pathology to establish a common metric of laminar distributions of pathology, thereby allowing direct comparisons of FTLD groups known to differ in total pathologic burden [22]. Ratios of layer pathology were measured in heterogeneous regions from distinct pathologic and clinical subgroups of FTLD and compared to WM pathology, disease severity, and cognitive impairment to elucidate patterns of potential progression and clinical relevance. Our comparative investigation indicates that TDP-43 pathology is consistently upper layer-predominant among TDP-43 proteinopathies, while tau pathology is more often lower layer-predominant among tauopathies, independent of region or clinical presentation. Furthermore, we provide new evidence that tau and TDP-43 pathology form different laminar distributions in the same dementia phenotypes and that upper layer pathology may contribute to global cognitive and behavioral deficits. These signature laminar patterns in separate FTLD proteinopathies may reveal distinct mechanisms of neurodegeneration in future work.

\section{Materials and methods}

\section{Patients}

Patients were clinically followed at the Frontotemporal Degeneration Center, Alzheimer's Disease Research Center, Comprehensive Amyotrophic Lateral Sclerosis Center, or Movement Disorder Center at the University of Pennsylvania (Penn). Clinical diagnoses were designated prospectively based on published consensus guidelines [10, 31, 40, 61, 82]. Patients evaluated prior to modern clinical criteria were assigned clinical phenotypes based on retrospective chart review and consensus by experienced clinicians and researchers (M.G., D.J.I., C.T.M., L.M., K.R., D.A.W.). Brain autopsies were performed at the Hospital of University of Pennsylvania and the 
Penn Center for Neurodegenerative Disease Research. As described previously [95], fresh tissue was sampled at autopsy and fixed overnight in $10 \%$ neutral buffered formalin or $70 \%$ ethanol with $150 \mathrm{mmol} \mathrm{NaCl}$. Neuropathologic diagnoses were made by neuropathologists (E.B.L. and J.Q.T.) using established criteria [22, 57, 60, 66]. All procedures in this study were performed in accordance with the standards of the Penn Institutional Review Board and the Declaration of Helsinki. Patient data were retrieved from the Penn Integrated Neurodegenerative Disease Database [103].

The current study included patients with a primary neuropathological diagnosis of either FTLD-tau $(n=73)$ or FTLD-TDP $(n=97)$. The FTLD-tau group included PiD $(n=14), \mathrm{CBD}(n=14)$, PSP $(n=36)$, and unclassifiable tauopathies (TauU, $n=9$ ), the latter included individuals with either unique tau accumulations $(n=3)$ or MAPT mutations $(n=6)$. The FTLD-TDP group included FTLDTDP types A $(n=25), \mathrm{B}(n=17), \mathrm{C}(n=17)$, and $\mathrm{E}(n=5)$, in addition to amyotrophic lateral sclerosis (ALS, $n=34$ ) comprising patients with motor-predominant ALS $(n=28)$ or ALS with FTD $(n=6)$. Patients were prospectively genotyped for pathogenic mutations on MAPT, C9orf72, $G R N$, and other FTD-associated genes based on a structured pedigree analysis described previously [102]. Carriers of C9orf72 $(n=21), G R N(n=14), T B K 1 \quad(n=2)$, and GBE1 ( $n=1)$ mutations met standard criteria for FTLDTDP subtype classification [57]. Some FTLD patients had secondary pathologic diagnoses that largely involved brain regions outside the cortical areas examined in the current study. Patient demographics and pathologic characteristics are summarized in Table 1.

\section{Clinical data}

To obtain a harmonized global measure of cognitive impairment available in the majority of our cohort spanning decades of brain banking, we focused on Mini-Mental State Examination (MMSE) scores collected from Penn INDD [24]. The relationship between laminar pathology and MMSE performance was tested using cross-sectional MMSE scores closest to death (within 4 years), which were available from a majority of FTLD-tau $(n=40)$ and FTLD-TDP $(n=56)$ patients. Total MMSE score ranges from unimpaired (30) to severely impaired (0) and used as a continuous measure in analyses below. To explore clinicopathologic relationships further, we compared laminar distributions of pathology to a validated measure of the severity of behavioral disturbances based on the Neuropsychiatric Inventory Questionnaire (NPI-Q) $[23,46]$. Cross-sectional NPI-Q data closest to death (within 4 years) were available in a subset of our cohort (13 FTLD-tau, 15 FTLD-TDP).

\section{Isocortical regions of interest}

The current investigation quantified laminar and WM pathology in ten isocortical regions (Fig. 1). The frequency of all regions examined in FTLD groups and subgroups are summarized in Supplementary Table 1 (online resource) including bilateral regions available from select frontal, temporal, and parietal regions in 33 brain autopsies. Functionally, we examined regions that belong to either idiotypic (i.e., M1 and V1) or association cortices, the latter referring to regions inside (i.e., aCING and aINS) or outside (i.e., aOFC, MFC, aITC, SMTC, pIPC) the paralimbic zone that subserve different cognitive and behavioral functions [62, 63]. Structurally, these regions have laminar organizations categorized as either agranular cortex (absent or inconspicuous layer IV) or granular/eulaminate cortex (six distinct layers or more) as summarized in Fig. $1[14,26]$.

Previous investigations of select tauopathies (i.e., PiD, PSP) and TDP-43 proteinopathies (i.e., ALS, FTLD-TDP types A-C) have identified regional patterns of tau and TDP43 pathology that may reflect stages of pathologic propagation between connected regions [18-20, 43, 50]. Thus, to examine patterns of potential pathologic progression across layers, we compared ratios of layer pathology between regions classified by their stage of involvement (earlier vs. later involved designations determined previously) [18-20, $43,50]$ or severity of total GM pathology (i.e., severe vs. mild designations determined by a region's median burden falling above or below the median burden of the total pathologic subgroup) (Supplementary Tables 2-3, online resource).

Given that multiple regions comprise distinguishable neural networks differentially involved in clinical syndromes such as bvFTD and PPA, we examined laminar patterns in regions part of the paralimbic salience network (bilateral aCING and aINS), the executive-control network (bilateral MFC and pSPC), and the language network (left MFC, aITC, SMTC, and pIPC) [76, 85, 89, 91, 92].

\section{Immunohistochemistry}

Immunohistochemistry procedures for regions sampled above were completed in the Penn Digital Neuropathology Lab and described in detail previously [29, 72]. In brief, paraffin-embedded $6 \mu \mathrm{m}$-thick sections were immunostained for either phosphorylated tau (mouse monoclonal antibody, pTau (S202/T205), clone AT8, 1:1000, Invitrogen) in the FTLD-tau group [17, 22] or phosphorylated TDP-43 (rat monoclonal antibody, pTDP-43 (S409/410), clone 1D3, 1:1000, MilliporeSigma) in the FTLD-TDP group [69]. All immunostained tissue was counterstained with fresh hematoxylin for the visualization of cell organization (i.e., cell density, morphology, and size), providing a reliable means 


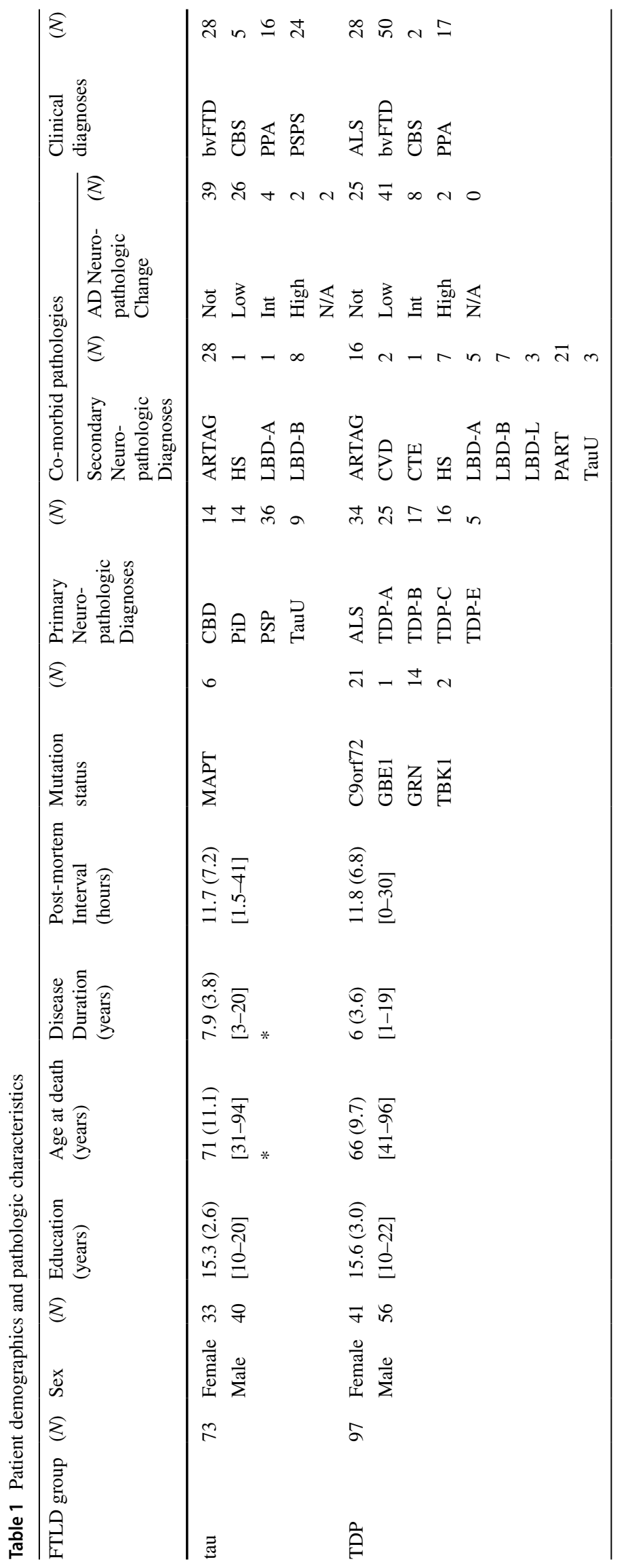




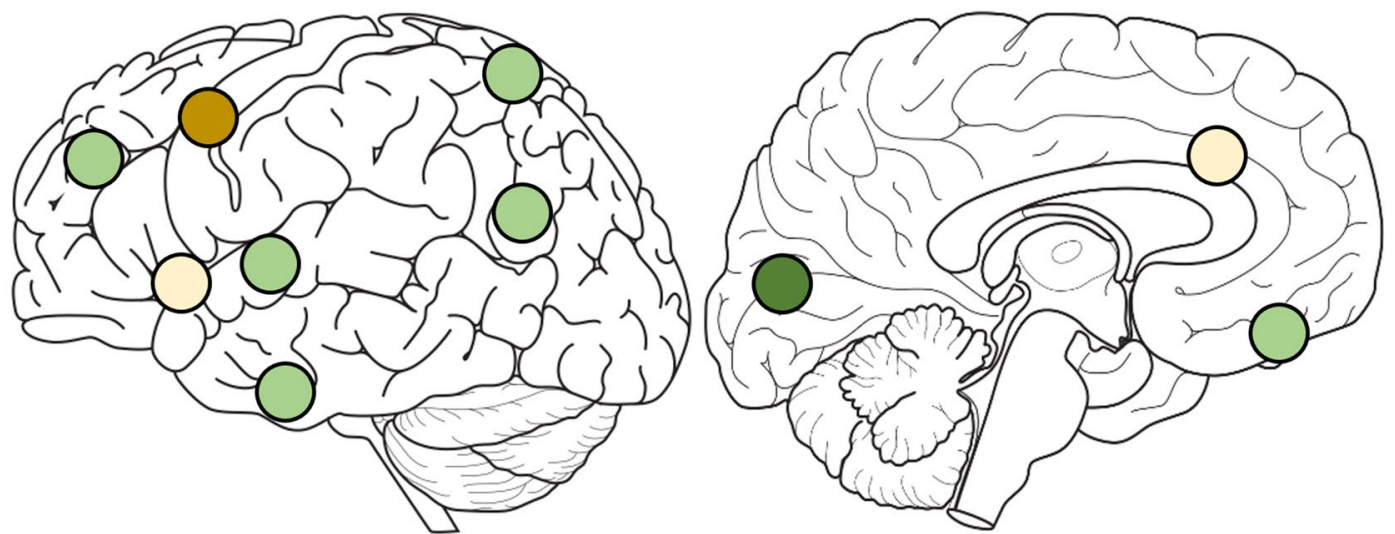

Figure Legend

\begin{tabular}{|c|c|c|c|c|c|}
\hline Lobe & Isocortical Region & & $\begin{array}{l}\text { Brodmann } \\
\text { Area }\end{array}$ & $\begin{array}{l}\text { Functional } \\
\text { type }\end{array}$ & $\begin{array}{l}\text { Structural } \\
\text { type }\end{array}$ \\
\hline \multirow{5}{*}{ Frontal } & primary motor cortex & M1 & 4 & Idiotypic & \multirow{3}{*}{ Agranular } \\
\hline & anterior cingulate cortex & aCING & 24 & \multirow{2}{*}{$\begin{array}{l}\text { Paralimbic } \\
\text { Association }\end{array}$} & \\
\hline & anterior insular cortex & aINS & 13 & & \\
\hline & anterior orbital frontal cortex & aOFC & 11 & \multirow{6}{*}{ Association } & \multirow{7}{*}{ Granular } \\
\hline & middle frontal cortex & MFC & 9 & & \\
\hline \multirow{2}{*}{ Temporal } & anterior inferior temporal cortex & aITC & 20 & & \\
\hline & superior/middle temporal cortex & SMTC & $22 / 21$ & & \\
\hline \multirow{2}{*}{ Parietal } & posterior inferior parietal cortex & pIPC & 39 & & \\
\hline & posterior superior parietal cortex & pSPC & 7 & & \\
\hline Occipital & primary visual cortex & V1 & 17 & Idiotypic & \\
\hline
\end{tabular}

Fig. 1 Isocortical regions of interest. Laminar distributions of pathology were quantified in ten isocortical regions associated with different lobes, functions, and structural cytoarchitecture. Agranular cortices included paralimbic association regions and idiotypic primary

to identify boundaries between cortical layers and WM in all regions (see details below).

\section{Laminar regions of interest and digital image approach}

Immunostained tissue sections were imaged on a digital slide scanner (Aperio AT2, Leica Biosystem, Wetzlar, Germany) in the Penn Digital Neuropathology Lab at $20 \times$ magnification. Images were digitally analyzed using QuPath software (version 0.2.0). Pathologic burden was measured as the percent area occupied (\% AO) by tau- or TDP-43-immunoreactive pixels in three subregions per section. Positive pixel classifiers were empirically derived for each batch motor cortex (dark brown). Granular cortices included association regions (light green) and more distinct granular cortex (koniocortex) included idiotypic primary visual cortex (dark green)

of immunostained sections in FTLD-tau and FTLD-TDP as previously validated [28].

An experienced neuroanatomist (D.T.O.) delineated the three subregions per section (i.e., upper layers I-III, lower layers IV-VI, and juxtacortical WM) based on regional cytoarchitecture (Supplementary Fig. 1, online resource). An unbiased view of the cytoarchitecture blinded to any immunoreactive inclusions that may be present in the field of view was created in QuPath by closing all color channels except the blue hematoxylin channel. Next, using a belt-transect method of sampling in each section [3, 44], three neighboring subregions orthogonal to the pial surface and $\sim 1 \mathrm{~mm}$ wide were manually annotated in the longest stretch of relatively flat isocortex (typically sulcal walls). 
This systematic sampling of gray matter avoided poorly represented cortical layers due to cortical folds or oblique cuts through gyri [84, 99].

Upper and lower layer subregions were differentiated according to laminar patterns of cellular organization (i.e., cellular size and density) specific to each isocortical region. For agranular regions with little to no layer IV (M1, aCING, and aINS), upper and lower layers were distinguishable by the relatively smaller pyramidal neurons of layer III abutting the larger pyramidal neurons of layer V. For granular/ eulaminate regions, the border between upper and lower layers was reliably identified by the relatively lower density of larger, mostly pyramidal neurons of layer III adjacent to the higher density of smaller, mostly non-pyramidal neurons of layer IV. In all regions, the border between layer VI and WM was consistently determined by the distinctly larger polymorphic neurons of layer VI adjacent to the higher density of smaller oligodendrocytes comprising WM. We excluded tissue with artifacts or tears that prevented the examination of cytoarchitecture and segmentation of all cortical laminae. In total, 1083 tissue sections met inclusion criteria for laminar analyses in the current study (Supplementary Table 1, online resource).

\section{Statistical analyses}

Total gray matter (GM) pathologic burden was measured as the average $\% \mathrm{AO}$ by pathology in combined cortical layers. We calculated ratios of upper to lower layer pathology and ratios of GM-to-WM pathology per section to preserve intrinsic pathologic distributions per patient and for direct comparisons between FTLD-tau and FTLD-TDP groups. Ratios of GM-to-WM pathology included the total GM pathologic burden in all cortical layers combined. GM pathology, WM pathology, and ratios of pathology underwent natural $\log (\ln )$ transformations to normalize data used in all analyses. The normalized layer ratio data produced positive values (upper layer-predominant pathology), negative values (lower layer-predominant pathology), or ratios approximately zero (bilaminar pathology). The normalized GM-to-WM ratio data produced positive or negative values that represented GM-predominant or WM-predominant pathology, respectively.

Demographics were compared using Chi-square analyses for categorical data and Wilcoxon Mann-Whitney U tests for non-parametric continuous data. Laminar distributions of pathology in FTLD-tau and FTLD-TDP were analyzed using linear mixed-effects (LME) models that included ratio of layer pathology as the dependent variable and random intercepts for individual patients to account for repeated measures and missing data. All LME models included hemisphere, region, age at death, and disease duration as fixed-effects and covariates to adjust for their potential contributions to the dependent variable (i.e., total GM pathology, ratio of layer pathology, or ratio of GM-to-WM pathology). Select analyses included additional fixed-effects and covariates as indicated below. Missing data due to tissue unavailability or poor integrity were accounted for in all models and results. All statistical tests were 2-sided and $p<0.05$ was considered significant. All analyses were performed using SPSS (version 27.0; SPSS, Chicago, IL).

\section{Comparisons of laminar pathology between FTLD groups and subgroups}

First, a LME model compared ratios of layer pathology by FTLD group (i.e., FTLD-tau and FTLD-TDP). To ensure concomitant pathologies did not account for differences in laminar pathology, analyses were repeated excluding patients with co-occurring pathologies. To confirm consistency of ratios of layer pathology within each FTLD group, pathologic subgroup (i.e., PiD, CBD, PSP, TauU in FTLDtau; TDP A-E, ALS in FTLD-TDP) was first included as a fixed-effect in separate LME models for each FTLD group. Second, genetic subgroup (i.e., sporadic vs. familial) was included as a fixed-effect in separate LME models for each FTLD group. Third, clinical subgroup (i.e., bvFTD, PPA, CBS, PSPS, ALS) was included as a fixed-effect in separate LME models for each FTLD group, with Bonferroni corrected pair-wise post hoc comparisons of main effects using estimated marginal means.

\section{Comparisons of laminar pathology between FTLD-tau and FTLD-TDP in select regions}

To confirm variations in regional microstructure and function did not account for differences in laminar pathology, ratios of layer pathology between total FTLD-tau and FTLDTDP were compared using three LME models in regions grouped by idiotypic M1, paralimbic association, or association cortex. Idiotypic V1 was a unilaterally sampled control region, thus linear regression compared the ratio of layer pathology in V1 to total FTLD-tau and FTLD-TDP, controlling for age at death and disease duration.

\section{Assessments of hypothesized progression of laminar pathology in FTLD-tau and FTLD-TDP}

Hypothesized disease progression across regions was tested using separate LME models for each FTLD group that included severe/mild pathology regions as a fixed-effect and total GM pathology as the dependent variable. These models were repeated using earlier/later involved regions as a fixed-effect and total GM pathology as the dependent variable. Next, separate LME models for each total FTLD group compared ratios of layer pathology between severe/ 


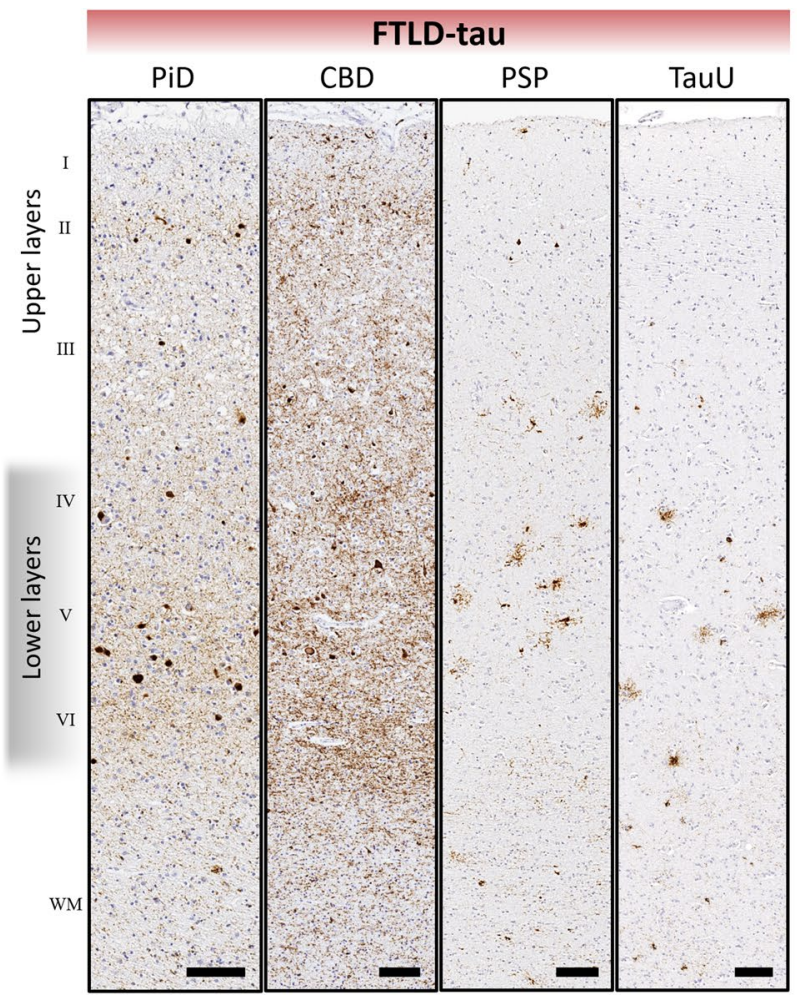

Fig. 2 Representative distributions of tau and TDP-43 pathology in cortical layers and juxtacortical white matter in pathologic subgroups of FTLD-tau and FTLD-TDP. FTLD-tau typically displayed a lower layer-predominant profile of AT8-immunoreactive tau pathology, while FTLD-TDP displayed an upper layer-predominant profile of 1D3-immunoreactive TDP-43 pathology. Neuronal and glial inclusions were interspersed among cortical layers and consistent with each pathologic subtype. In FTLD-tau, neuronal inclusions (i.e., pretangles, tangles, globose tangles, ballooned neurons) and glial inclusions (i.e., tufted astrocytes, astrocytic plaques, ramified astrocytes, coiled bodies) were found in all cortical layers in most regions exam-

mild pathology regions. Similarly, separate LME models for each total FTLD group compared ratios of GM-to-WM pathology between severe/mild pathology regions. These analyses were repeated in earlier/later involved regions for ratios of layer pathology and ratios of GM-to-WM pathology in each FTLD group. Finally, separate LME models for each total FTLD group tested ratios of layer pathology as a function of juxtacortical WM pathology.

\section{Relationships between laminar pathology and clinical assessments of cognition and behavior in FTLD-tau and FTLD-TDP}

First, separate LME models for each total FTLD group tested total GM pathology as a function of the continuous variable final MMSE score. Next, separate LME models for each total FTLD group tested ratios of layer pathology as a

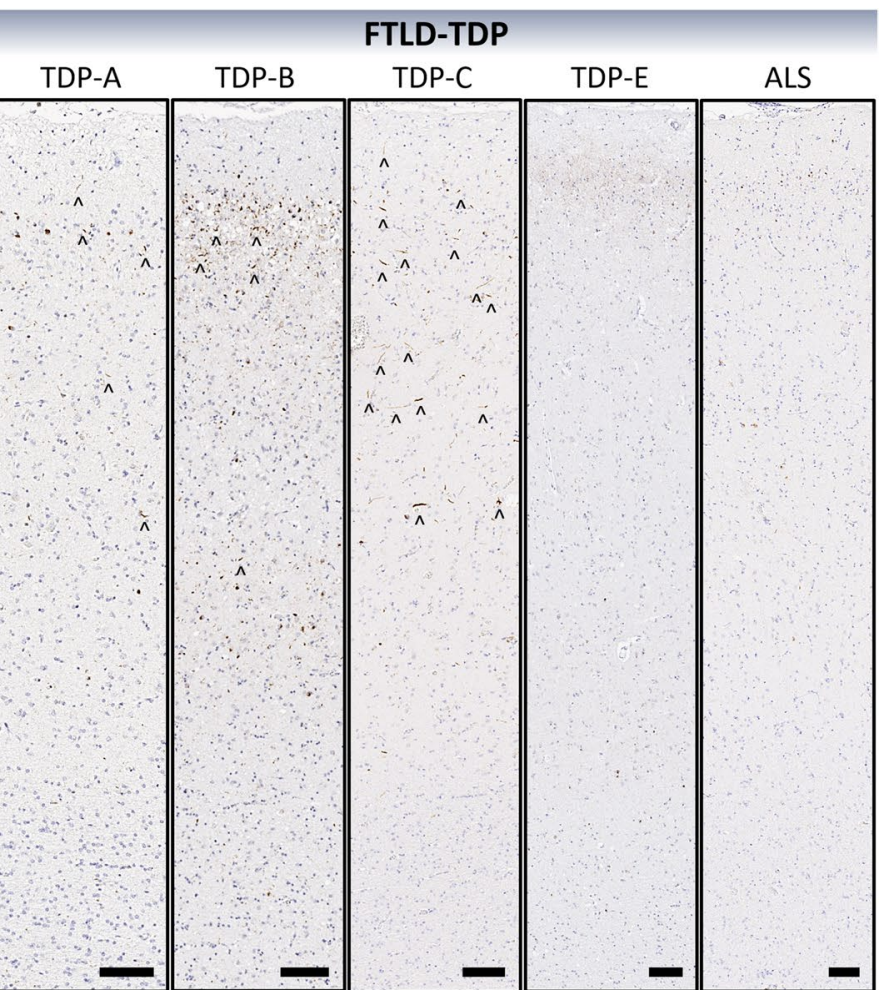

ined, with high densities of neuritic threads common to lower layers in many regions. WM tau accumulation was often similar to total GM tau burden, and generally resembled neuritic threads or coiled bodies. In FTLD-TDP, TDP-43 pathology was observed more often in upper layers and was comprised of mostly small neuronal cytoplasmic inclusions and dystrophic neurites (open arrows point to examples of small neurites) depending on the pathologic subtype of TDP. TDP-43 pathology in WM typically resembled curvilinear oligodendrocytic inclusions that were consistently less frequent than TDP-43 pathology in GM. All images acquired from the MFC region. Roman numerals indicate cortical layers. Scale bars $=100 \mu \mathrm{m}$

function of final MMSE score or final NPI-Q score. Finally, given that the MMSE may better detect cognitive impairment in bvFTD compared to other non-amnestic phenotypes [73], separate LME models for bvFTD-tau and bvFTD-TDP tested ratios of layer pathology as a function of final MMSE score (fixed-effect). All LME models that examined clinicopathologic relationships included the following fixedeffects/covariates: hemisphere, region, sex, education, age at death, and time interval between final clinical assessment and death.

\section{Comparisons of laminar pathology between FTLD-tau and FTLD-TDP in bvFTD}

We repeated analyses of ratios of layer pathology in bvFTD and PPA syndromes caused by changes to regions part of distinct cognitive networks. First, separate LME models for 


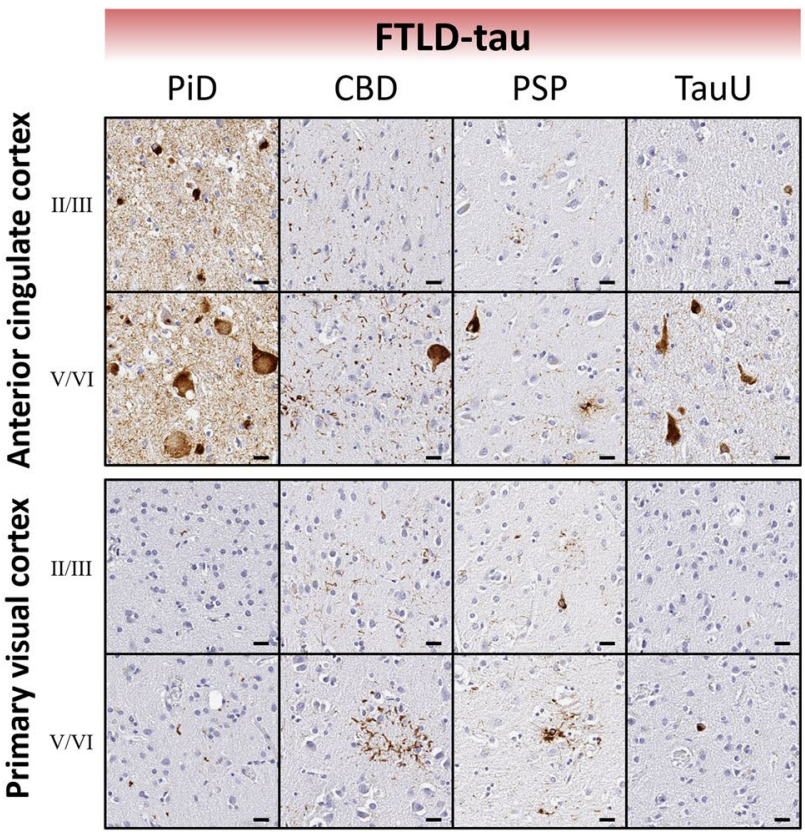

Fig. 3 Representative photomicrographs of tau and TDP-43 pathology in supragranular and infragranular layers of select regions. Distinct laminar distributions of tau and TDP-43 pathology were largely

bvFTD and PPA compared ratios of layer pathology between FTLD-tau and FTLD-TDP. Second, we accounted for clinically relevant regions in each clinical phenotype in the following models: one LME model compared ratios of layer pathology between FTLD-tau and FTLD-TDP in bilateral salience/executive-related regions relevant to bvFTD, and another LME model compared ratios of layer pathology between FTLD-tau and FTLD-TDP in left language-related regions relevant to PPA.

\section{Results}

\section{Patient demographics}

FTLD groups were well-matched across multiple demographics and pathologic features (Table 1). On average, the total FTLD-tau group was older $(Z=-3.14, p=0.002)$ and had a longer disease duration $(Z=-2.91, p=0.004)$ than the total FTLD-TDP group.

\section{Distinct laminar distributions of pathology in FTLD-tau and FTLD-TDP}

TDP-43 pathology was consistently observed in upper layers with relatively less involvement of deeper cortex across

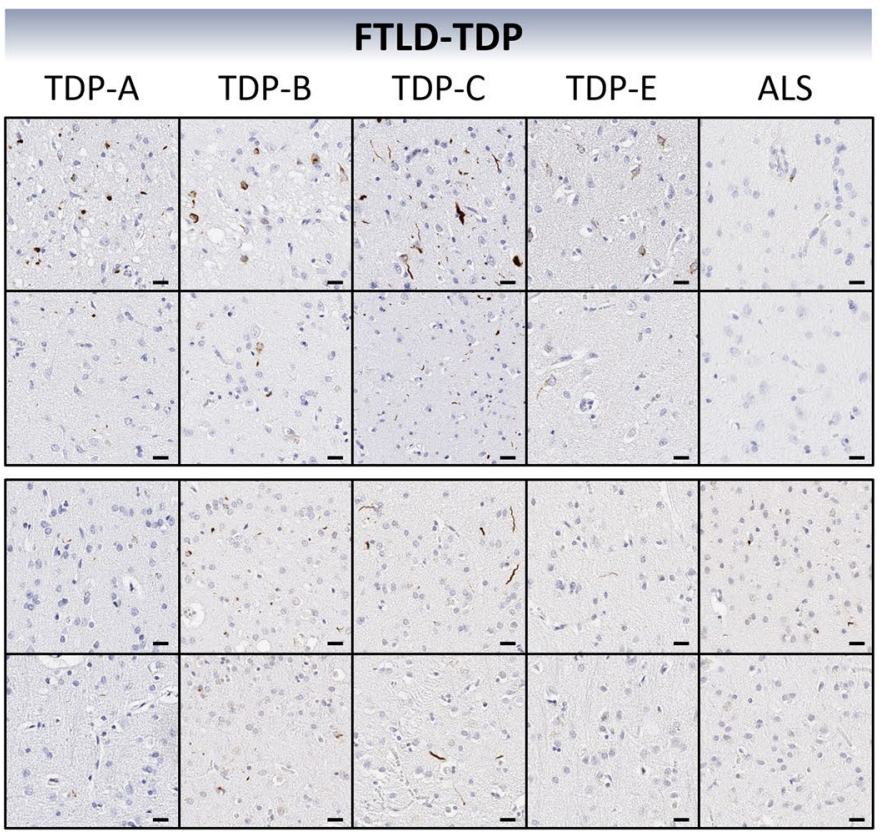

consistent across regions examined, despite regional differences in cytoarchitecture, function, or total pathologic burden. Roman numerals indicate cortical layers. Scale bars $=20 \mu \mathrm{m}$

FTLD-TDP subgroups. In contrast, tau pathology was more frequent and widespread across cortical layers, with prominent involvement of lower layers and WM across FTLD-tau subgroups (Figs. 2, 3). To quantitatively determine if tau and TDP-43 pathology accumulated unique patterns across cortical laminae, we first compared ratios of layer pathology between total FTLD-tau and FTLD-TDP groups. We found that FTLD-tau was associated with a significantly lower ratio of layer pathology compared to the higher ratio of layer pathology in FTLD-TDP $(\beta=-0.62, \mathrm{SE}=0.07, p<0.001)$, indicating relatively greater tau burden in lower layers compared to relatively greater TDP-43 burden in upper layers (Fig. 4a). In this model, all other effects were non-significant $(p>0.7)$ except region $(p=0.003)$.

We replicated these findings in a subset of patients with no missing data (i.e., complete data from the same frontal or temporal regions), suggesting that variations in region availability (missing data) between patients did not influence the protienopathy-specific laminar distributions of pathology we measured (Supplementary Fig. 3, online resource). Importantly, we found similar results when we excluded the ALS subgroup $(n=34)$ from the total FTLD-TDP group $(n=97)$, leaving only patients with TDP types A-E $(n=63)$. Specifically, we found that FTLD-tau still displays relatively greater lower layer pathology in comparison to the relatively greater upper layer pathology in clinically similar TDP types A-E $(\beta=-0.65, \mathrm{SE}=0.07, p<0.001)$. 

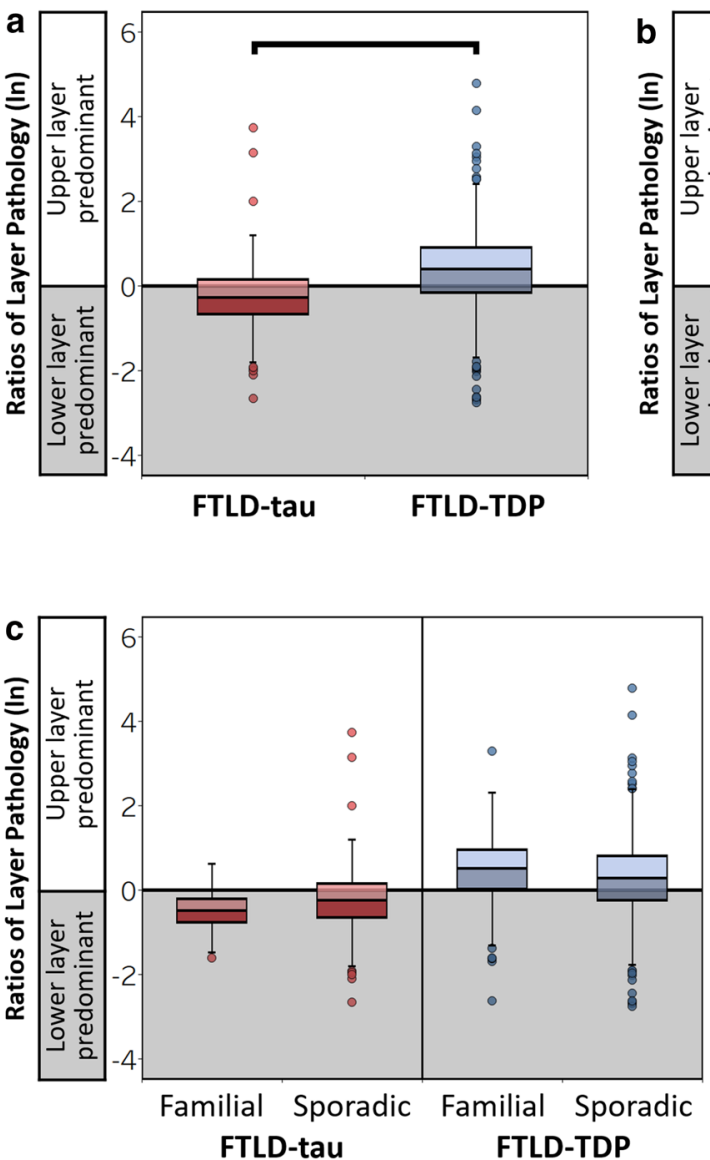

Fig. 4 Laminar distributions of tau and TDP-43 pathology are distinct and consistent between pathologic, genetic, and clinical subgroups. Ratios of layer pathology by total FTLD groups (a). FTLDtau was associated with a significantly lower ratio of layer pathology (lower layer-predominant tau pathology) compared to the higher ratio of larger pathology (upper layer-predominant TDP-43 pathology) in

Next, we tested for consistency between pathologic, genetic, and clinical subgroups within FTLD-tau and FTLDTDP (Fig. 4b-d, Supplementary Table 4, online resource). Pathologic subgroups of FTLD-tau displayed similarly low ratios of layer pathology $(p=0.132)$ and pathologic subgroups of FTLD-TDP displayed similarly high ratios of layer pathology $(p=0.265)$ (Fig. 4b). Ratios of layer pathology did not differ between sporadic and familial subgroups of FTLD-tau ( $p=0.637)$ or FTLD-TDP $(p=0.267)$ (Fig. 4c). Lastly, we found that ratios of layer pathology were largely consistent between clinical subgroups of FTLDTDP $(p=0.540)$ and between clinical subgroups FTLD-tau (Fig. 4d, Supplementary Table 4, online resource).

To confirm that laminar patterns were not influenced by concomitant pathology (Table 1), we excluded patients with any pathology secondary to FTLD-tau and FTLD-TDP, including low-to-high AD neuropathologic change (ADNC). Similar to total group results, we found lower ratios of layer

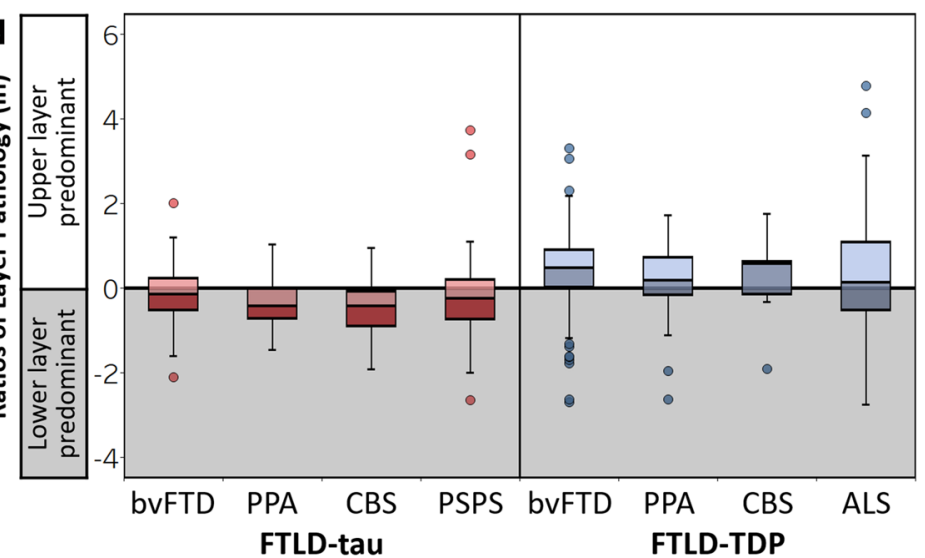

FTLD-TDP $(\beta=-0.61, \mathrm{SE}=0.07, p<0.001)$. Within-group comparisons of ratios of layer pathology by pathologic (b), genetic (c), and clinical subgroups (d) demonstrate that distinct ratios of layer pathology are largely similar between subgroups of FTLD-tau and between subgroups of FTLD-TDP. Boxplots represent ratios of layer pathology per region of each patient

pathology in relatively "pure" FTLD-tau $(n=22)$ compared to "pure" FTLD-TDP $(n=22 ; \beta=-0.60, \mathrm{SE}=0.08$, $p<0.001)$ while all other effects were non-significant $(p>0.2)$.

\section{Distinct laminar distributions of pathology in FTLD-tau and FTLD-TDP are consistent across regions with varied cytoarchitecture and function}

We performed a series of regional analyses to determine if ratios of layer pathology differed by regions with different microstructure or function. In total FTLD groups, we analyzed regional subgroups based on microstructural and functional affiliations and consistently found distinct ratios of layer pathology between FTLD-tau and FTLDTDP in idiotypic M1 $(\beta=-0.34, \mathrm{SE}=0.12, p=0.004)$, paralimbic association $(\beta=-0.81, \mathrm{SE}=0.19, p<0.001)$, 


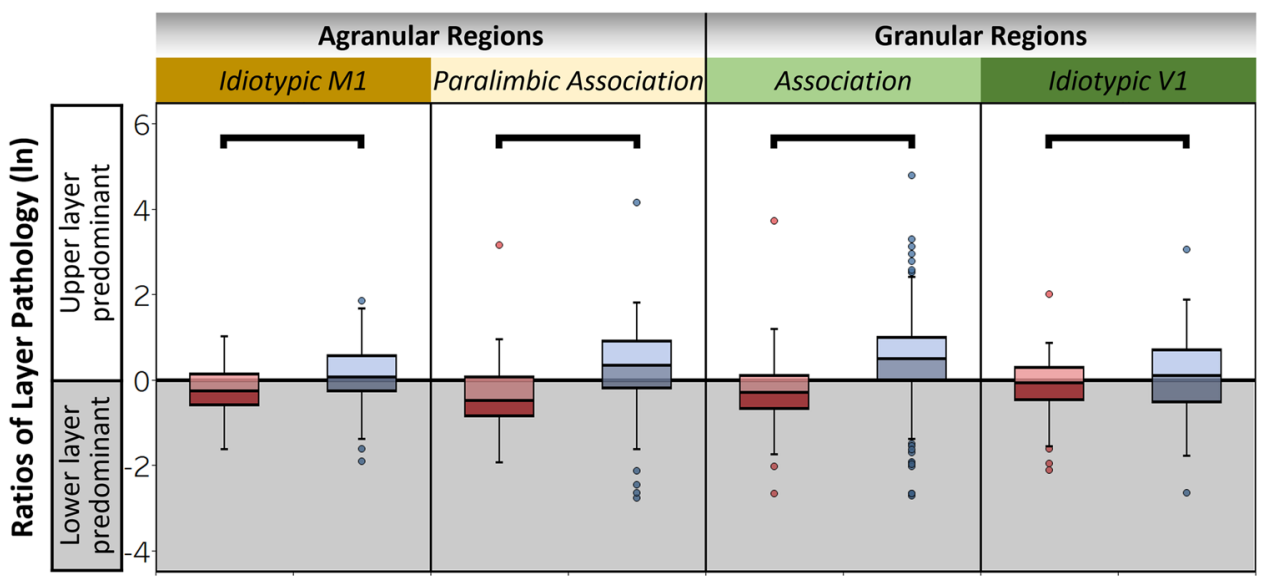

FTLD-tau FTLD-TDP FTLD-tau FTLD-TDP FTLD-tau FTLD-TDP FTLD-tau FTLD-TDP

Fig. 5 Distinct laminar distributions of tau and TDP-43 pathology are consistent across regions with varied cytoarchitecture and function. Ratios of layer pathology in regions with varied microstructure and function. Total FTLD-tau and total FTLD-TDP groups displayed significant differences in ratios of layer pathology in each type of agran-

association $(\beta=-0.72, \mathrm{SE}=0.08, p<0.001)$, and idiotypic $\mathrm{V} 1(\beta=0.30, \mathrm{SE}=0.14, p=0.037)$ (Fig. 5). In these models, we observed a significant effect of region in paralimbic association regions $(p=0.047)$, while all other effects were nonsignificant $(p>0.05)$. These regional comparisons provide converging evidence that laminar distributions of pathology appear relatively unique to each proteinopathy across heterogeneous regions sampled.

\section{Laminar patterns of pathology in relation to regional disease severity and juxtacortical white matter in FTLD-tau and FTLD-TDP}

Because regions of different disease severity/stage of involvement may reflect patterns of pathologic spread [18-20, 43, 50], we tested the hypothesis that ratios of layer pathology may differ between these regions in FTLD-tau and FTLD-TDP. As expected, regions categorized as earlier involved or severe pathology had significantly greater total GM pathology compared to later involved or mild pathology regions, confirming that these approaches to categorizing regions may reflect disease progression (Supplementary Table 2-3, Supplementary Fig. 2a-b, online resources). Next, we compared ratios of layers pathology between regions categorized as severe or mild pathologic burden and found lower ratios of layer pathology in severe pathology regions compared to mild pathology regions $(\beta=-0.20$, $\mathrm{SE}=0.07, p=0.007$ ) in FTLD-tau (Fig. 6a) while all other effects were non-significant $(p>0.07)$. In contrast, relatively high ratios of layer pathology were not different between severe pathology and mild pathology regions $(\beta=-0.09$, $\mathrm{SE}=0.10, p=0.362$ ) in FTLD-TDP (Fig. 6a), indicating ular and granular region examined, including idiotypic M1 $(\beta=-0.34$, $\mathrm{SE}=0.12, p=0.004)$, paralimbic association regions $(\beta=-0.81$, $\mathrm{SE}=0.19, \quad p<0.001)$, association regions $(\beta=-0.71, \mathrm{SE}=0.08$, $p<0.001)$, and idiotypic V1 $(\beta=-0.29, \mathrm{SE}=0.14, p=0.037)$. Boxplots represent ratios of layer pathology per region of each patient

that TDP-43 pathology is largely restricted to upper layers throughout disease progression.

Similar to above, we tested the hypothesis that total GM pathology and laminar distributions of pathology may be related to juxtacortical WM pathology due to potential spread of pathology between the connected regions. In analyses of FTLD-tau, we found lower GM-to-WM ratios of tau pathology (greater WM-predominance) in severe pathology regions compared to mild pathology regions $(\beta=-0.34, \mathrm{SE}=0.10, p=0.001)$ while all other effects were non-significant $(p>0.09)$ except region $(p=0.021)$ (Fig. 6b). Additionally, greater WM tau pathology was associated with lower ratios of layer tau pathology $(\beta=-0.05, \mathrm{SE}=0.02, p=0.002)$, while all other effects were non-significant $(p>0.3)$ except region $(p=0.048)$ (Fig. 6c). Analyses of FTLD-TDP produced distinct results from FTLD-tau: high ratios of GM-to-WM pathology (greater GM predominance) were not different between severe and mild pathology regions $(\beta=0.18, \mathrm{SE}=0.14$, $p=0.199$ ) (Fig. 6b), and WM TDP-43 pathology did not relate to ratios of layer TDP-43 pathology $(\beta=-0.00006$, $\mathrm{SE}=0.02, p=0.998$ ) in FTLD-TDP (Fig. 6c). Subanalyses controlling for pathologic subgroup produced similar findings. In FTLD-tau, pathologic subgroup was not a significant predictor $(p=0.081)$ and it did not influence the significant relationship between WM pathology and the ratio of layer tau pathology $(\beta=-0.06, \mathrm{SE}=0.02, p<0.001)$. Similarly, in FTLD-TDP, pathologic subgroup was not a significant predictor $(p=0.210)$ and it did not influence the lack of relationship between WM pathology and the ratio of layer TDP-43 pathology $(\beta=-0.02, \mathrm{SE}=0.03$, $p=0.370)$. Alternative categorization of regions into 
Fig. 6 Laminar distributions of pathology in relation to regional disease severity and juxtacortical white matter in FTLD-tau and FTLDTDP. (a) Ratios of layer pathology by regions with severe or mild pathologic burden in total FTLD-tau and FTLD-TDP. In FTLD-tau, severe pathology regions displayed lower ratios of tau pathology compared to mild pathology regions $(\beta=-0.20, \mathrm{SE}=0.07, p=0.006)$. In FTLD-TDP, however, higher ratios of layer pathology were similar between mild and severe pathology regions $(\beta=-0.10, \mathrm{SE}=0.10$, $p=0.309$ ). (b) Ratios of GM-to-WM pathology by regions with severe or mild pathologic burden in total FTLD-tau and FTLD-TDP. In FTLD-tau, severe pathology regions displayed lower ratios of GMto-WM pathology compared to mild pathology regions $(\beta=-0.34$, $\mathrm{SE}=0.10, p=0.001)$. In FTLD-TDP, however, similar higher ratios of GM-to-WM pathology were displayed in severe and mild pathology regions $(\beta=0.17, \mathrm{SE}=0.14, p=0.228)$. (c) Greater $\mathrm{WM}$ tau pathology was associated with lower ratios of layer pathology in FTLD-tau $(\beta=-0.05, \mathrm{SE}=0.02, p=0.001)$, whereas there was no relationship between WM TDP-43 pathology and the ratio of layer pathology in FTLD-TDP $(\beta=-0.003, \mathrm{SE}=0.02, p=0.90)$. Lines represent the predicted ratios of layer pathology as a function of WM pathology controlling for covariates in each model (i.e., hemisphere, region, age at death, and disease duration). Boxplots and scatterplots represent ratios of layer pathology per region of each patient

earlier vs. later stages of involvement produced similar results (Supplementary Fig. 3, online resource). These analyses provide converging evidence that TDP-43 pathology is consistently upper layer-predominant, whereas tau pathology is more pronounced in the lower layers and WM of severe pathology regions potentially earlier involved in disease progression.

\section{Clinicopathologic relationships in FTLD-tau and FTLD-TDP}

We explored the relationships between laminar pathology and global cognitive impairment in FTLD patients evaluated closest to death $(n=96)$. First, we found that lower MMSE scores were associated with greater total GM pathology in FTLD-tau $(\beta=-0.08, \mathrm{SE}=0.02, p=0.003)$; all other effects were non-significant effects $(p>0.1)$ except region $(p<0.001)$ and age at death $(p=0.002)$. Similarly, lower MMSE scores were associated with greater total GM pathology in FTLD-TDP $(\beta=-0.10, \mathrm{SE}=0.02, p<0.001)$; all other effects were non-significant $(p>0.1)$ except region $(p<0.001)$ (Fig. 7a). Despite these relationships, we found no relationship between MMSE score and the upper layer-predominant pathology characteristic of FTLD-TDP $(\beta=0.001, \mathrm{SE}=0.01, p=0.838)$. In contrast, lower MMSE score was associated with higher ratios of layer pathology in FTLD-tau ( $\beta=-0.02, \mathrm{SE}=0.01, p=0.018)$ (Fig. 7b); all other effects were non-significant $(p>0.05)$ except region $(p=0.001)$. Next, we performed a focused subanalysis in bvFTD $(n=44)$ to avoid potential phenotypic differences in cognitive testing [73]. Similar to total FTLD groups, we found no relationship between the ratio of layer pathology and MMSE score in bvFTD-TDP $(\beta=0.003, \mathrm{SE}=0.01$, a

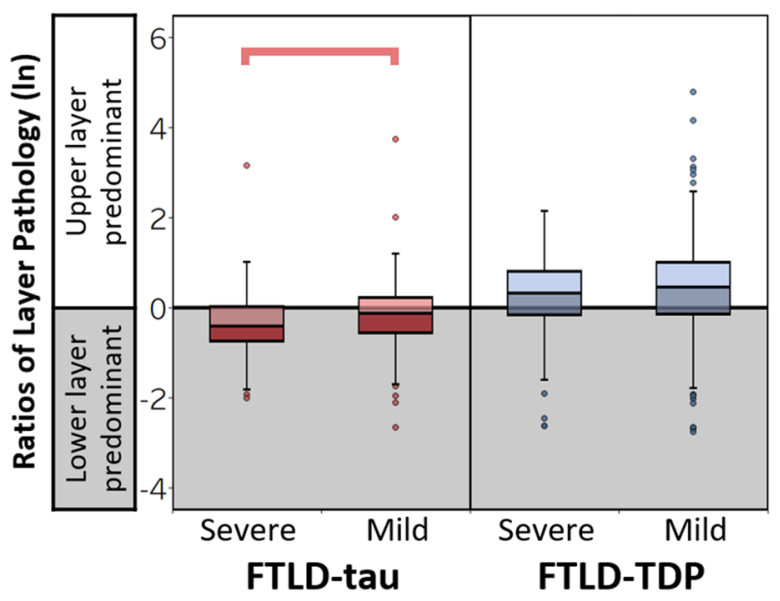

b

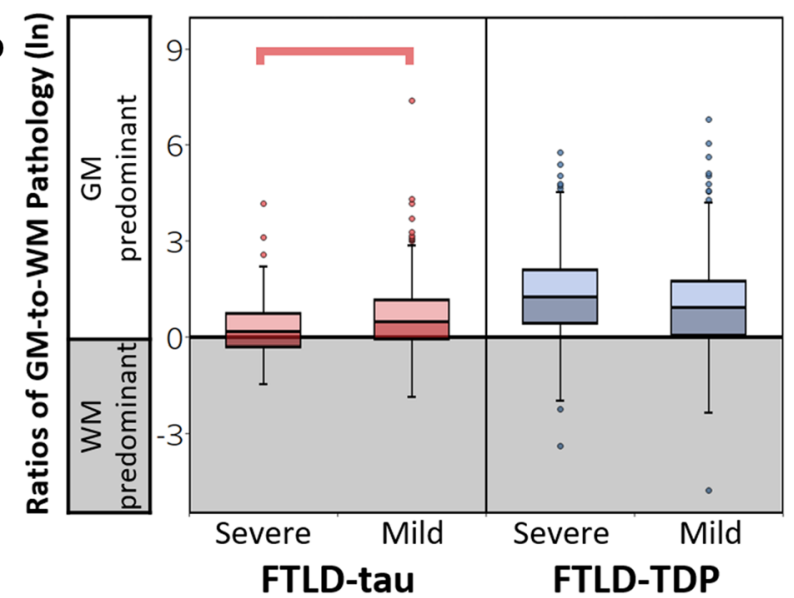

c

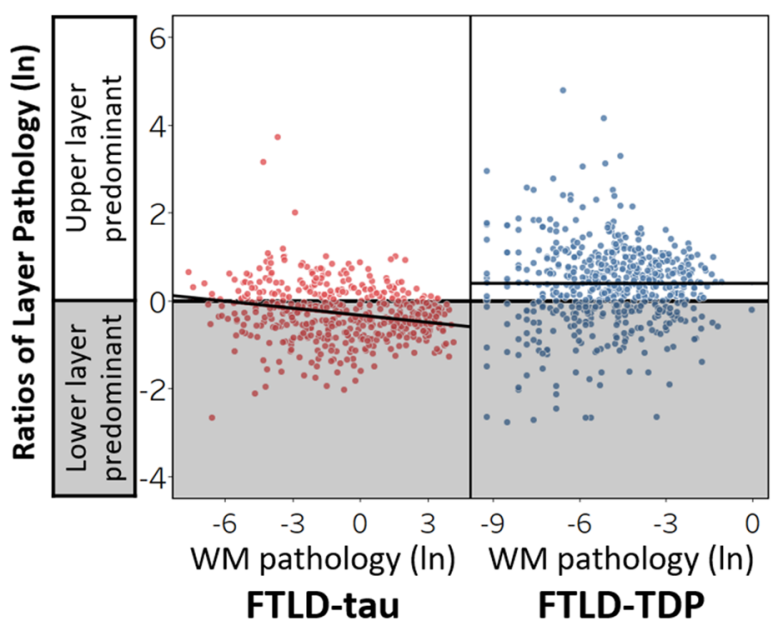

$p=0.752$ ), whereas lower MMSE score was associated with higher ratios of layer pathology in bvFTD-tau $(\beta=-0.04$, $\mathrm{SE}=0.01, p=0.004)$. In a subset of our total FTLD cohort with behavioral changes measured close to death by the NPI-Q, we found that greater upper layer-predominant tau pathology was related to more behavioral disturbances in the total FTLD-tau group, but not the total FTLD-TDP group (Supplementary Fig. 6, online resource). These findings 




Fig. 7 Relationships between laminar distributions of pathology and global cognitive impairment in FTLD-tau and FTLD-TDP. (a) Lower MMSE score was associated with greater total gray matter (GM) pathology in both FTLD-tau $(\beta=-0.08, \mathrm{SE}=0.02, p=0.003)$ and FTLD-TDP $(\beta=-0.10, \mathrm{SE}=0.02, p<0.001)$. Lines represent the predicted total GM pathology as a function of final MMSE controlling for covariates in each model (i.e., hemisphere, region, sex, education, age at death, and time interval between final MMSE and death). (b) Lower MMSE score was associated with higher ratios of

from the NPI-Q, in combination with MMSE data, provide converging evidence that a disproportionate accumulation of pathology in upper layers may contribute to cognitive and behavioral deficits in clinically similar FTLD-tau and FTLD-TDP patients.

\section{FTLD-tau and FTLD-TDP display signature laminar distributions of pathology in bvFTD and PPA}

We tested whether distinct clinical syndromes and regions clinically relevant to these syndromes predict similar laminar distributions of pathology by examining our largest dementia subgroups, bvFTD and PPA. Consistent with total group results, we first found lower ratios of layer pathology in FTLD-tau compared to FTLD-TDP in both bvFTD $(\beta=-0.50, \mathrm{SE}=0.09, p<0.001)$ and PPA $(\beta=-0.73$, $\mathrm{SE}=0.14, p<0.001)$ with a significant effect of region in each model $(p<0.04)$. Next, we repeated these analyses in regions clinically relevant to bvFTD and PPA. In bilateral salience/executive-related regions implicated in bvFTD, we found a lower ratio of layer pathology in bvFTD-tau compared to bvFTD-TDP $(\beta=-0.63, \mathrm{SE}=0.12, p<0.001)$ (Fig. 8a); all other effects were non-significant $(p>0.2)$. Similarly, in left language regions underlying PPA, we found a lower ratio of layer pathology in PPA-tau compared to PPA-TDP ( $\beta=-0.46, \mathrm{SE}=0.21, p=0.046)$ (Fig. $8 b$ ); all other effects were non-significant $(p>0.4)$.

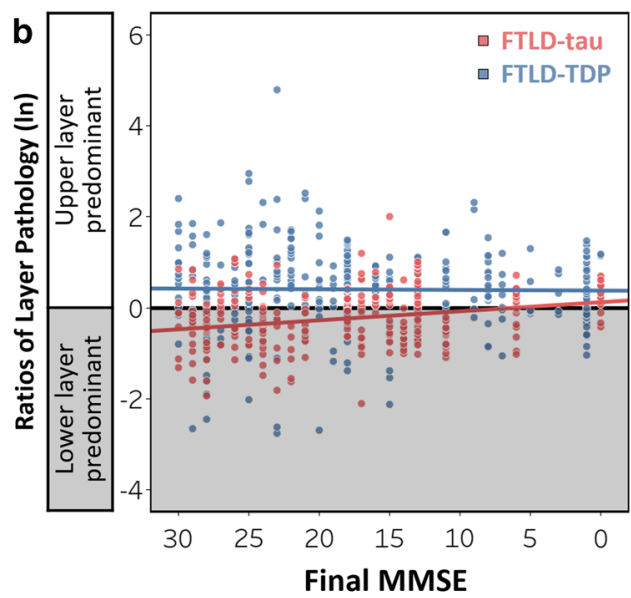

layer pathology in FTLD-tau $(\beta=-0.02, \mathrm{SE}=0.01, p=0.018)$. In contrast, there was no relationship between MMSE score and the ratio of layer pathology in FTLD-TDP $(\beta=0.001, \mathrm{SE}=0.01, p=0.838)$. Lines represent the predicted ratios of layer pathology as a function of final MMSE controlling for covariates in each model (i.e., hemisphere, region, sex, education, age at death, and time interval between final MMSE and death). Scatterplots represent ratios of layer pathology per region of each patient

\section{Discussion}

This comparative study finds that FTLD tauopathies feature relatively greater tau burden in lower layers and WM, while TDP-43 proteinopathies display relatively greater TDP-43 burden in upper layers with minimal WM involvement. Distinct laminar distributions of pathology were specific to proteinopathy and largely consistent across the isocortex and FTLD subgroups, providing new evidence that clinically relevant regions and clinically distinct subgroups are not significant predictors of laminar changes. These data support the hypothesis that prominent lower layer changes help distinguish FTLD-tau from FTLD-TDP. As a result, tau and TDP-43 may not affect the same cells and neural circuits that interconnect regions, despite the evidence for similar large-scale dysfunction/degeneration of clinically relevant networks in FTD-FTLD patients. Therefore, pathologic changes to layer-specific neurons and their predictable feedforward/feedback projections may inform models of neurodegenerative disease and guide the development of biomarkers sensitive to laminar pathologic change.

AD-type neurofibrillary tangles accumulate in mostly cortical layers III and V of clinical AD patients, forming a well-documented bilaminar distribution $[11,16,54,75]$. However, laminar changes in FTD patients are far less established. Previous examinations have focused on only select inclusions in small cohorts of neuropathologically defined FTLD with limited, if any, clinical comparisons. By accounting for demographic factors, regions sampled, 
a
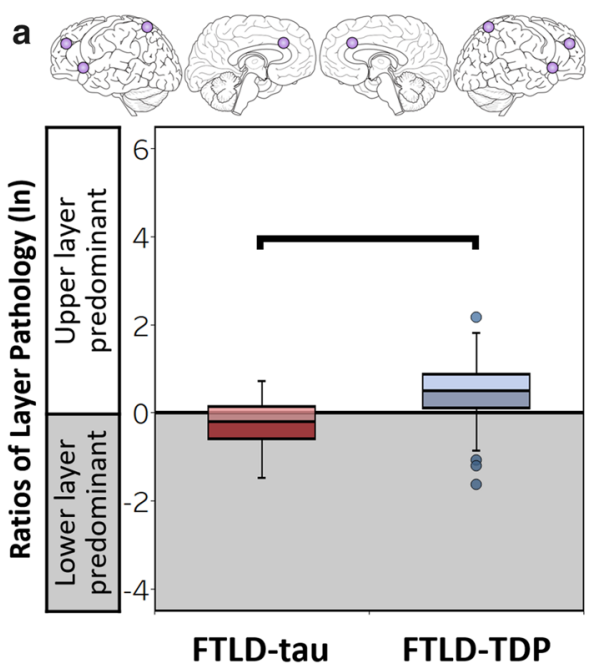

C
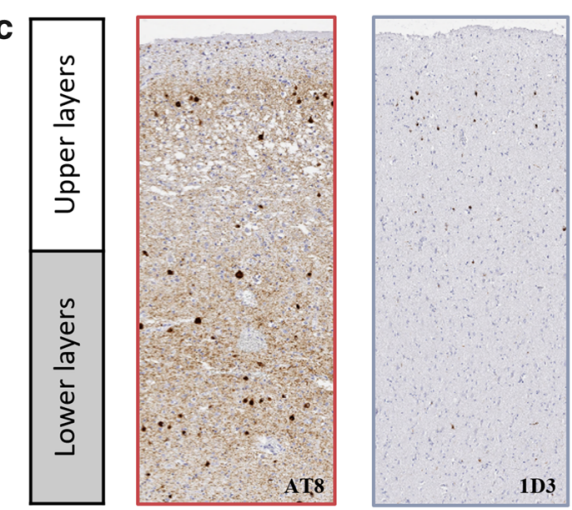

Fig. 8 FTLD-tau and FTLD-TDP display signature laminar distributions of pathology in bvFTD and PPA. (a) Ratios of layer pathology in bilateral regions clinically relevant to bvFTD (i.e., aCING, aINS, MFC, pSPC). Bilateral salience/executive-related regions displayed lower ratios of layer pathology in bvFTD-tau compared to bvFTD$\operatorname{TDP}(\beta=-0.63, \mathrm{SE}=0.12, p<0.001)$. (b) Ratios of layer pathology in left hemisphere regions clinically relevant to PPA (i.e., MFC, aITC, SMTC, pIPC). Left language-related regions displayed lower ratios of layer pathology in PPA-tau compared to PPA-TDP $(\beta=-0.49$,

and subgroup classifications, our large comparative study of digitally measured pathologic burden addresses the limitations of prior studies to advance our understanding of the unique aggregation of FTLD pathologies in heterogeneous FTD syndromes. Despite known pathologic subtypes and distinct clinical phenotypes, we find that lower layer-predominant tau pathology is a common feature of FTLD-tau and upper layer-predominant TDP-43 pathology is a unifying pattern in FTLD-TDP (Fig. 2-4). Graphical representations of pathology appeared bilaminar in select subgroups (Fig. 4), but analyses comparing PiD, ALS, and total FTLD groups suggest that PiD and ALS still have distinct patterns (data not shown). In PiD, tau burden may appear bilaminar due to frequent Pick body accumulation in upper layers and prominent Pick cells in lower layers [5, 38, 43]. In ALS

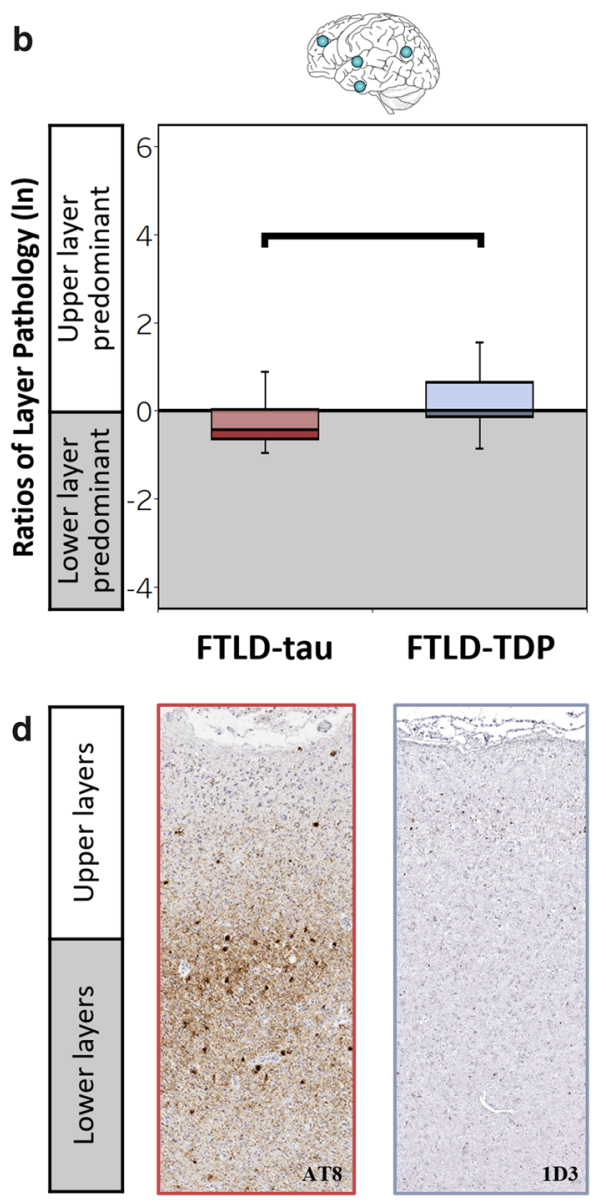

$\mathrm{SE}=0.09, p<0.001)$. (c) Representative images of lower layerpredominant AT8-immunoreactive tau pathology (left) and upper layer-predominant 1D3-immunoreactive TDP-43 pathology (right) in the aCING region of bvFTD patients with either PiD (left) or TDP-A (right). (d) Representative images of lower layer-predominant AT8-immunoreactive tau pathology (left) and upper-predominant 1D3-immunoreactive TDP-43 pathology (right) in the left SMTC region of PPA patients with either PiD (left) or TDP-A (right)

and TDP subtypes B-E, multiple types of TDP-43 inclusions are known to accumulate in both upper and lower layers, contributing to subtype classification criteria $[19,20$, $53,56,58]$. However, our layer ratios of total pathologic burden likely capture threads and other small pathologies incompletely assessed by previous studies, producing novel results that indicate that TDP-43 burden is distinctly greater in upper layers even when infrequent lower layer inclusions are present in various FTLD-TDP subtypes. In contrast, tau burden has a predilection for lower layers, regardless of upper layer involvement or type of tauopathy.

Neurodegenerative diseases such as AD often co-occur with FTLD [83], and select studies of PiD, CBD, PSP, and ALS with concomitant AD have found that AD-type tangles accumulate more often in supragranular layers II-III 
as opposed to infragranular layers V-VI [5, 34, 37-39]. Co-pathologies were relatively infrequent in our FTLD cohorts, but when present, were mostly restricted to regions outside the isocortex including limbic areas, subcortex, and brainstem (Table 1). To address the possibility that laminar distributions of FTLD pathology are altered by co-morbid pathology, we excluded FTLD brains with known co-morbid pathology, including low-to-high ADNC. In the remaining "pure" FTLD brains, we replicated our main findings of lower layer-predominant pathology in FTLD-tau and upper layer-predominant pathology in FTLD-TDP. While we cannot rule out a contribution of mixed pathology on laminar patterns in FTLD, co-morbid pathology appeared to have little effect on differences observed between FTLD-tau and FTLD-TDP.

Our large regional investigation teased apart the potential effects of regional variations in microstructure and function on laminar pathology. We demonstrated that unique laminar distributions of pathology associated with FTLD-tau and FTLD-TDP were largely consistent across regions with specialized functions (i.e., idiotypic, association, and paralimbic association regions) and intrinsic variations in laminar cytoarchitecture (e.g., agranular versus granular cortex) (Fig. 4). Region was often a significant predictor of laminar pathology in our between-group models, suggesting that we cannot entirely rule out region-specific contributions to laminar profiles in FTLD. Future investigations of the cells occupying each cortical layer will help determine which layer-specific cell populations are most vulnerable to degeneration among these diverse but interconnected regions in FTLD.

Pathologic proteins including FTLD-type tau and TDP43 may seed and propagate via synaptically connected brain regions $[25,30,52,78]$. Postmortem human tissue cannot directly track the temporal sequence of cell-to-cell transmission, but known laminar circuits represent a neuroanatomical model useful for testing predictions made by transneuronal spread hypotheses [26]. Specifically, distinct laminar profiles of FTLD pathology may indirectly point to which layer-specific neurons are influencing spread of pathology into connected cortices and into juxtacortical WM comprised of both efferents exiting from and afferents arriving to cortical layers. Accordingly, the current study compared laminar pathology to neighboring WM and between interconnected regions with varied disease severity. We classified regional disease severity using total GM pathologic burden and histopathologic staging data in FTLD [18-20, 43, 50] (Supplementary Tables $2-3$, online resource), permitting the comparison of laminar distributions of pathology between regions with mild pathology (later involved) and severe pathology (earlier involved). In FTLD-tau, we found that severe pathology/earlier involved regions displayed greater tau pathology in lower layers and WM compared to mild pathology/later involved regions (Fig. 6, Supplementary
Fig. 3, online resource). Furthermore, greater WM tau pathology was related to relatively greater lower layer tau pathology (Fig. 6c). One interpretation of these results is that select regions are vulnerable to early and preferential tau accumulation in lower layers and WM before tau spreads into later involved regions with bilaminar susceptibilities to tau deposits. Alternatively, initial tau distributions may be bilaminar, but there is selective pathologic accumulation in and progressive degeneration of lower layers, especially in the earliest involved regions that accrue the most severe pathologic burden. Indeed, a previous study of CBD showed that earlier onset and shorter disease duration were associated with bimodal/bilaminar distributions of tau pathology [7]. While we cannot rule out the unique contributions of high pathologic burden in WM in select pathologic subgroups such as CBD, pathologic subgroup was not a significant predictor in models that adjusted for subgroups (data not shown). Taken together, tau pathology may have a predilection for WM fibers due in part to layer-specific neurons (especially infragranular neurons) disproportionately targeted by tau pathology.

Pathologic spread of TDP-43 pathology may not follow the same laminar pathways as tau pathology according to our regional analyses in FTLD-TDP. Specifically, we found that laminar distributions of pathology were not associated with WM pathology and TDP-43 remained upper layer-predominant between regions of different pathologic severity or stage of involvement (Fig. 6, Supplementary Fig. 2, online resource). Consistent with a previous study in FTLD-TDP [9], these data may reflect the propensity of TDP-43 proteinopathies to accumulate and spread among upper layers compared to lower layers and WM. However, more bilaminar distributions of TDP-43 pathology have been shown to be related to increasing disease duration [9], a pattern we did not observe possibly due in part to contrasting methodologic designs. Indeed, our current study measured all immunoreactive inclusions in carefully delineated layers in a large diverse FTLD-TDP cohort as opposed to the previous study that only quantified a subset of TDP-43 inclusions without delineating cortical layers in a smaller FTLDTDP cohort. The anatomical pathways by which pathology can spread are many, but the regional laminar patterns of pathology observed here provide preliminary evidence that FTLD-tau and FTLD-TDP may spread via different neural circuits. Upper layer-predominant TDP-43 pathology suggests corticocortical circuits may be the primary mode of transmission between cortices throughout disease progression in FTLD-TDP. In contrast, widespread interlaminar tau suggests that more diverse circuits mediate tau spread in FTLD-tau, including infragranular pathways that uniquely project to both cortical and subcortical regions.

The histopathologic correlates of cognitive decline remain elusive in much of the FTLD spectrum. To address 
this, we compared laminar pathology to performance on the MMSE closest to death, a neuropsychological exam that measured the severity of global cognitive impairment in the majority of our cohort. First, we found greater pathology in total GM was related to greater cognitive impairment in both FTLD groups (Fig. 7a), which validates our digital measurements and supports previous work that suggests tau and TDP-43 aggregation are associated with dementia severity $[19,20,43,50,79]$. Second, we found a novel relationship in FTLD-tau such that greater cognitive impairment was associated with a higher ratio of layer pathology corresponding to bilaminar/upper layer-predominant tau burden (Fig. 7b). These results were replicated in our largest dementia subgroup bvFTD $(n=44)$ to account for potential confounds of clinical phenotype on testing [73]. In contrast to FTLD-tau, we found no relationship between the ratio of layer pathology and MMSE performance in total FTLD-TDP or bvFTDTDP. Interestingly, we found similar results in both FTLDtau and FTLD-TDP when we compared laminar pathology to the severity of behavioral disturbances as measured by the NPI-Q [23, 46] (Supplementary Fig. 6, online resource). One explanation for these findings is that global cognitive and behavioral impairment may be closer related to upper layer degeneration than lower layer degeneration, regardless of the underlying FTLD proteinopathy. Thus, a common feature of clinically similar FTLD-tau and FTLD-TDP patients (e.g., bvFTD) may be the degeneration of supragranular corticocortical circuits that connect numerous higher association cortices [84]. Indeed, a common clinical impairment in bvFTD is disinhibition, a behavior recently linked to aberrant neural activity potentially mediated by supragranular circuits in the frontal cortex of bvFTD patients [41]. We had limited harmonized clinical data near end stage of disease for our clinicopathologic analyses. While we carefully accounted for time interval between clinical testing and autopsy in our models and restricted analyses to data collected close to death, shorter time intervals are an inherent challenge to retrospective clinicopathologic studies. However, these data are important as there is currently no neuroimaging modality capable of diagnosing FTLD neuropathology during life (especially at the resolution of cortical layers), necessitating our present postmortem investigation. Therefore, while two global clinical scales (MMSE and NPI-Q) produced converging evidence that laminar distributions of pathology may influence clinical features of FTLD patients, these findings remain exploratory. Further evaluation is needed in future investigations with more detailed and consistent longitudinal assessments of cognition and behavior as they become available.

Our comparative study included a diverse range of clinical phenotypes to determine whether laminar pathology is related to FTD presentations underrepresented or missed in previous laminar studies. Of note was the inclusion of
bvFTD and PPA, our two largest clinical dementia subgroups that have a similar prevalence of underlying tauopathies or TDP-43 proteinopathies. We show for the first time that regardless if patients presented with bvFTD or PPA, tauopathies had a common lower layer-predominant profile of pathology whereas TDP-43 proteinopathies had a characteristic upper layer-predominant profile of pathology. It is important to note that laminar distributions of tau and TDP-43 pathology remained distinguishable even when our analyses were restricted to regions clinically relevant to bvFTD (bilateral salience/executive-related regions) [76, 87, $89,91,92,104]$ and PPA (left language-related regions) [64, 85, 86] (Fig. 8). Due to the rarity of the semantic variant of PPA with FTLD-tau and the non-fluent variant of PPA with FTLD-TDP [27, 65, 94], we were unable to test for differences between these subgroups in our current cohort. Divergent laminar distributions of FTLD pathology in bvFTD or PPA hold biological and clinical significance given the anatomy of cortical layer connectivity. Namely, supragranular layers are enriched for neurons with corticocortical connections, while lower layers have a mix of neurons that connect a range of cortical, subcortical, brainstem, and thalamic structures [14, 84, 93]. These neural circuits all make diverse connections with larger cognitive networks implicated in FTD syndromes in ways not well understood. However, it is conceivable that pathologic changes to separate cortical layers and regions may converge onto a common network downstream that leads to clinically indistinguishable presentations [21, 101, 104]. Given the proteinopathy-specific distributions of pathology across cortical layers observed here, selective laminar neurodegeneration may influence the clinical profile via distinct anatomical pathways. Thus, pathologic changes to select cortical layers may inform the neural substrates of cognition and behavior in FTD, and if detected during life, could improve diagnoses and guide new disease-modifying approaches for FTLD patients.

The present study included nine FTLD proteinopathies and five clinical dementia or motor syndromes to advance our understanding of laminar pathology in the heterogeneous populations of FTLD. We calculated ratios of layer pathology to preserve the relative distribution within a region and individual patient and to permit the comparison of disparate proteinopathies independent of variability in overall burden. However, this investigation was not without some limitations. Some of our pathologic and clinical subgroups were relatively small due in part to the rarity of FTLD, requiring future studies to include multicenter participation to replicate and generalize our findings. While we sampled up to ten different anatomical regions, there were missing data for some regions due to varied tissue integrity, exhausted tissue, or evolution of brain sampling from our brain bank over the past 35 years. Linear mixed-effects models accounted for missing data, but more 
complete regional sampling will be performed in prospective autopsies. Given that the current study sampled one section per region available, more expansive sampling of regions using a larger series of tissue or thicker sections will be important to future laminar studies. Lastly, new examinations of laminar neurodegeneration will further elucidate the laminar patterns of disease in FTLD as reliable methods are developed and validated.

In conclusion, our results suggest that laminar distributions of pathology are relatively specific to proteinopathies, with an upper layer-predominant profile in FTLDTDP compared to a lower layer-predominant profile in FTLD-tau. Furthermore, we find that patients with the same bvFTD or PPA clinical syndromes still display distinct laminar profiles of tau and TDP-43 pathology, even within regions that contribute to the network dysfunction underlying the syndromes. Therefore, macroscopic abnormalities in networks may have dissimilar microscopic or mesoscopic underpinnings based on the involvement of select cortical layers differentially targeted by FTLD-tau and FTLD-TDP. Given the predictable connections to and from cortical layers, it follows that specific feedforward and feedback circuits may contribute to patterns of propagation, neurodegeneration, and clinical presentations in ways not currently understood in FTD-FTLD patients. Microscopic analyses of cortical layers provide an advantageous anatomical framework for mapping and understanding the local cellular effects of FTLD proteinopathy on macroscopic network degeneration and clinical symptomology [76, 77, 101]. Moreover, laminar determinants of clinical features in FTD may inform antemortem biomarkers of proteinopathy, especially with the use of highresolution MRI measures of cortical layers and connectivity under development $[12,51,96,100]$.

Supplementary Information The online version contains supplementary material available at https://doi.org/10.1007/s00401-021-02402-3.

Acknowledgements We greatly appreciate the technical assistance provided by John Robinson, Theresa Schuck, Winifred Trotman, Emily Xie, and Rebecca Lobrovich. We also thank the patients and families for their invaluable contributions and participation in the brain donation program that made this study possible.

Author contributions All authors contributed to the interpretation of the work and approved the submitted manuscript. DTO and DJI contributed to the design, data acquisition, analyses, interpretation, drafting, and revision of the work. CP, SXX, and KAQC contributed to data acquisition and analyses. DAW, VVD, LE, MS, and AD contributed to data acquisition. CTM, JQT, EBL, MG contributed to the data acquisition, interpretation, and revising of the work.

Funding This work was supported by NIH grants NINDS R01NS109260-01A1, NIA P01-AG066597, NIA P30-AG072979 (forme rly P30-AG10124), NIA R01-AG054519-02, NIA R01-AG03849001A1, NIA U01-AG052943-01, NIA U19-AG062418-03, Penn
Institute on Aging, the Wyncote Foundation, and former NINDS P50-NS053488-09, NIA P01-AG017586 and NIA P01-AG032953.

Data availability The datasets collected and/or analyzed during the current study are available from the corresponding author on reasonable request.

\section{Declarations}

Conflict of interest EBL and JQT are members of Acta Neuropathologica's Editorial Board but were not involved in the editorial handling of this article.

Ethics approval All procedures performed in studies involving human participants were in accordance with the ethical standards of University of Pennsylvania Internal Review Board and with the 1964 Helsinki Declaration and its later amendments or comparable ethical standards. This article does not contain any studies with animals performed by any of the authors.

Informed consent Informed consent was obtained from all individual participants included in the study.

Open Access This article is licensed under a Creative Commons Attribution 4.0 International License, which permits use, sharing, adaptation, distribution and reproduction in any medium or format, as long as you give appropriate credit to the original author(s) and the source, provide a link to the Creative Commons licence, and indicate if changes were made. The images or other third party material in this article are included in the article's Creative Commons licence, unless indicated otherwise in a credit line to the material. If material is not included in the article's Creative Commons licence and your intended use is not permitted by statutory regulation or exceeds the permitted use, you will need to obtain permission directly from the copyright holder. To view a copy of this licence, visit http://creativecommons.org/licenses/by/4.0/.

\section{References}

1. Agosta F, Weiler M, Filippi M (2015) Propagation of pathology through brain networks in neurodegenerative diseases: from molecules to clinical phenotypes. CNS Neurosci Ther 21:754-767

2. Amunts K, Schleicher A, Zilles K (2007) Cytoarchitecture of the cerebral cortex-More than localization. Neuroimage 37:1061-1065

3. Armstrong RA (2003) Quantifying the pathology of neurodegenerative disorders: quantitative measurements, sampling strategies and data analysis. Histopathology 42:521-529

4. Armstrong RA, Cairns NJ (2009) Clustering and spatial correlations of the neuronal cytoplasmic inclusions, astrocytic plaques and ballooned neurons in corticobasal degeneration. J Neural Transm 116:1103-1110

5. Armstrong C, Lantos (1999) Laminar distribution of pick bodies, pick cells and Alzheimer disease pathology in the frontal and temporal cortex in Pick's disease. Neuropathol Appl Neurobiol 25:266-271

6. Armstrong RA, Cairns NJ, Lantos PL (1999) Clustering of cerebral cortical lesions in patients with corticobasal degeneration. Neurosci Lett 268:5-8

7. Armstrong RA, Lantos PL, Cairns NJ (2000) Laminar distribution of ballooned neurons and tau positive neurons with 
inclusions in patients with corticobasal degeneration. Neurosci Res Commun 27:85-93

8. Armstrong RA, Carter D, Cairns NJ (2012) A quantitative study of the neuropathology of 32 sporadic and familial cases of frontotemporal lobar degeneration with TDP-43 proteinopathy (FTLDTDP). Neuropathol Appl Neurobiol 38:25-38

9. Armstrong RA, Hamilton RL, Mackenzie IRA, Hedreen J, Cairns NJ (2013) Laminar distribution of the pathological changes in sporadic frontotemporal lobar degeneration with transactive response (TAR) DNA-binding protein of $43 \mathrm{kDa}$ (TDP-43) proteinopathy: a quantitative study using polynomial curve fitting. Neuropathol Appl Neurobiol 39:335-347

10. Armstrong MJ, Litvan I, Lang AE, Bak TH, Bhatia KP, Borroni $B$ et al (2013) Criteria for the diagnosis of corticobasal degeneration. Neurology 80:496-503

11. Arnold SE, Hyman BT, Flory J, Damasio AR, Hoesen GWV (1991) The topographical and neuroanatomical distribution of neurofibrillary tangles and neuritic plaques in the cerebral cortex of patients with Alzheimer's disease. Cereb Cortex 1:103-116

12. Assaf $Y$ (2019) Imaging laminar structures in the gray matter with diffusion MRI. Neuroimage 197:677-688

13. Barbas H (1986) Pattern in the laminar origin of corticocortical connections. J Comp Neurol 252:415-422

14. Barbas H (2015) General cortical and special prefrontal connections: principles from structure to function. Annu Rev Neurosci 38:1-21

15. Barbas H, Rempel-Clower N (1997) Cortical structure predicts the pattern of corticocortical connections. Cereb Cortex 7:635-646

16. Braak H, Braak E (1991) Neuropathological stageing of Alzheimer-related changes. Acta Neuropathol 82:239-259

17. Braak H, Alafuzoff I, Arzberger T, Kretzschmar H, Tredici KD (2006) Staging of Alzheimer disease-associated neurofibrillary pathology using paraffin sections and immunocytochemistry. Acta Neuropathol 112:389-404

18. Braak H, Brettschneider J, Ludolph AC, Lee VM, Trojanowski JQ, Tredici KD (2013) Amyotrophic lateral sclerosis-a model of corticofugal axonal spread. Nat Rev Neurol 9:708-714

19. Brettschneider J, Tredici KD, Toledo JB, Robinson JL, Irwin DJ, Grossman M et al (2013) Stages of pTDP-43 pathology in amyotrophic lateral sclerosis. Ann Neurol 74:20-38

20. Brettschneider J, Tredici KD, Irwin DJ, Grossman M, Robinson JL, Toledo JB et al (2014) Sequential distribution of pTDP43 pathology in behavioral variant frontotemporal dementia (bvFTD). Acta Neuropathol 127:423-439

21. Buckner RL, Sepulcre J, Talukdar T, Krienen FM, Liu H, Hedden T et al (2009) Cortical hubs revealed by intrinsic functional connectivity: mapping, assessment of stability, and relation to Alzheimer's disease. J Neurosci 29:1860-1873

22. Cairns NJ, Bigio EH, Mackenzie IRA, Neumann M, Lee VMY, Hatanpaa KJ et al (2007) Neuropathologic diagnostic and nosologic criteria for frontotemporal lobar degeneration: consensus of the Consortium for Frontotemporal Lobar Degeneration. Acta Neuropathol. https://doi.org/10.1007/s00401-007-0237-2

23. Cummings JL, Mega M, Gray K, Rosenberg-Thompson S, Carusi DA, Gornbein J (1994) The Neuropsychiatric Inventory: comprehensive assessment of psychopathology in dementia. Neurology 44:2308-2308

24. Folstein MF, Folstein SE, McHugh PR (1975) "Mini-mental state" a practical method for grading the cognitive state of patients for the clinician. J Psychiatr Res 12:189-198

25. Frost B, Diamond MI (2010) Prion-like mechanisms in neurodegenerative diseases. Nat Rev Neurosci 11:155-159

26. García-Cabezas MÁ, Zikopoulos B, Barbas H (2019) The Structural Model: a theory linking connections, plasticity, pathology, development and evolution of the cerebral cortex. Brain Struct Funct 224:985-1008

27. Giannini LAA, Xie SX, McMillan CT, Liang M, Williams A, Jester C et al (2019) Divergent patterns of TDP-43 and tau pathologies in primary progressive aphasia. Ann Neurol 85:630-643

28. Giannini LAA, Xie SX, Peterson C, Zhou C, Lee EB, Wolk DA et al (2019) Empiric methods to account for pre-analytical variability in digital histopathology in frontotemporal lobar degeneration. Front Neurosci 13:682

29. Giannini LAA, Peterson C, Ohm D, Xie SX, McMillan CT, Raskovsky K et al (2021) Frontotemporal lobar degeneration proteinopathies have disparate microscopic patterns of white and grey matter pathology. Acta Neuropathol Commun 9:30

30. Gibbons GS, Lee VMY, Trojanowski JQ (2019) Mechanisms of cell-to-cell transmission of pathological tau: a review. JAMA Neurol 76:101

31. Gorno-Tempini ML, Hillis AE, Weintraub S, Kertesz A, Mendez M, Cappa SF et al (2011) Classification of primary progressive aphasia and its variants. Neurology 76:1006-1014

32. Goulas A, Zilles K, Hilgetag CC (2018) Cortical gradients and laminar projections in mammals. Trends Neurosci 41:775-788

33. Goulas A, Changeux J-P, Wagstyl K, Amunts K, PalomeroGallagher N, Hilgetag CC (2021) The natural axis of transmitter receptor distribution in the human cerebral cortex. Proc Natl Acad Sci 118:e2020574118

34. Hauw JJ, Verny M, Delaère P, Cervera P, He Y, Duyckaerts C (1990) Constant neurofibrillary changes in the neocortex in progressive supranuclear palsy. Basic differences with Alzheimer's disease and aging. Neurosci Lett 119:182-186

35. Heuvel MP, Scholtens LH, Barrett LF, Hilgetag CC, Reus MA (2015) Bridging cytoarchitectonics and connectomics in human cerebral cortex. J Neurosci 35:13943-13948

36. Hilgetag CC, Beul SF, Albada SJV, Goulas A (2019) An architectonic type principle integrates macroscopic cortico-cortical connections with intrinsic cortical circuits of the primate brain. Netw Neurosci 3:905-923

37. Hof PR, Delacourte A, Bouras C (1992) Distribution of cortical neurofibrillary tangles in progressive supranuclear palsy: a quantitative analysis of six cases. Acta Neuropathol 84:45-51

38. Hof PR, Bouras C, Perl DP, Morrison JH (1994) Quantitative neuropathologic analysis of Pick's disease cases: cortical distribution of Pick bodies and coexistence with Alzheimer's disease. Acta Neuropathol 87:115-124

39. Hof PR, Perl DP, Loerzel AJ, Steele JC, Morrison JH (1994) Amyotrophic lateral sclerosis and parkinsonism-dementia from Guam: differences in neurofibrillary tangle distribution and density in the hippocampal formation and neocortex. Brain Res 650:107-116

40. Höglinger GU, Respondek G, Stamelou M, Kurz C, Josephs KA, Lang AE et al (2017) Clinical diagnosis of progressive supranuclear palsy: the movement disorder society criteria. Mov Disord 32:853-864

41. Hughes LE, Rittman T, Robbins TW, Rowe JB (2018) Reorganization of cortical oscillatory dynamics underlying disinhibition in frontotemporal dementia. Brain 141:2486-2499

42. Irwin DJ, Cairns NJ, Grossman M, McMillan CT, Lee EB, Deerlin VMV et al (2014) Frontotemporal lobar degeneration: defining phenotypic diversity through personalized medicine. Acta Neuropathol 129:469-491

43. Irwin DJ, Brettschneider J, McMillan CT, Cooper F, Olm C, Arnold SE et al (2016) Deep clinical and neuropathological phenotyping of Pick disease. Ann Neurol 79:272-287

44. Irwin DJ, Byrne MD, McMillan CT, Cooper F, Arnold SE, Lee EB et al (2016) Semi-automated digital image analysis of 
pick's disease and TDP-43 proteinopathy. J Histochem Cytochem 64:54-66

45. Irwin DJ, McMillan CT, Xie SX, Rascovsky K, Deerlin VMV, Coslett HB et al (2018) Asymmetry of post-mortem neuropathology in behavioural-variant frontotemporal dementia. Brain 141:288-301

46. Kaufer DI, Cummings JL, Ketchel P, Smith V, MacMillan A, Shelley T et al (2000) Validation of the NPI-Q, a brief clinical form of the neuropsychiatric inventory. J Neuropsychiatry Clin Neurosci 12:233-239

47. Kim E-J, Sidhu M, Gaus SE, Huang EJ, Hof PR, Miller BL et al (2012) Selective frontoinsular von economo neuron and fork cell loss in early behavioral variant frontotemporal dementia. Cereb Cortex 22:251-259

48. Kim G, Ahmadian SS, Peterson M, Parton Z, Memon R, Weintraub $S$ et al (2016) Asymmetric pathology in primary progressive aphasia with progranulin mutations and TDP inclusions. Neurology 86:627-636

49. Kim G, Bolbolan K, Shahidehpour R, Jamshidi P, Gefen T, Ayala IA et al (2019) Morphology and Distribution of TDP-43 Pre-inclusions in Primary Progressive Aphasia. J Neuropathol Exp Neurol 78:229-237

50. Kovacs GG, Lukic MJ, Irwin DJ, Arzberger T, Respondek G, Lee EB et al (2020) Distribution patterns of tau pathology in progressive supranuclear palsy. Acta Neuropathol 140:99-119

51. Lawrence SJD, Formisano E, Muckli L, Lange FPd (2019) Laminar fMRI: applications for cognitive neuroscience. Neuroimage 197:785-791

52. Lee S-J, Desplats P, Sigurdson C, Tsigelny I, Masliah E (2010) Cell-to-cell transmission of non-prion protein aggregates. Nat Rev Neurol 6:702-706

53. Lee EB, Porta S, Baer GM, Xu Y, Suh E, Kwong LK et al (2017) Expansion of the classification of FTLD-TDP: distinct pathology associated with rapidly progressive frontotemporal degeneration. Acta Neuropathol 134:65-78

54. Lewis DA, Campbell MJ, Terry RD, Morrison JH (1987) Laminar and regional distributions of neurofibrillary tangles and neuritic plaques in Alzheimer's disease: a quantitative study of visual and auditory cortices. J Neurosci 7:1799-1808

55. Lin L-C, Nana AL, Hepker M, Hwang J-HL, Gaus SE, Spina S et al (2019) Preferential tau aggregation in von Economo neurons and fork cells in frontotemporal lobar degeneration with specific MAPT variants. Acta Neuropathol Commun 7:159

56. Mackenzie IR, Neumann M (2017) Reappraisal of TDP-43 pathology in FTLD-U subtypes. Acta Neuropathol 134:79-96

57. Mackenzie IRA, Neumann M, Bigio EH, Cairns NJ, Alafuzoff I, Kril J et al (2009) Nomenclature for neuropathologic subtypes of frontotemporal lobar degeneration: consensus recommendations. Acta Neuropathol 117:15-18

58. Mackenzie IRA, Neumann M, Bigio EH, Cairns NJ, Alafuzoff I, Kril J et al (2010) Nomenclature and nosology for neuropathologic subtypes of frontotemporal lobar degeneration: an update. Acta Neuropathol 119:1-4

59. Marquié M, Normandin MD, Meltzer AC, Chong MST, Andrea NV, Antón-Fernández A et al (2017) Pathological correlations of [F-18]-AV-1451 imaging in non-alzheimer tauopathies: tau Imaging: pathological Correlations. Ann Neurol 81:117-128

60. McKeith IG, Boeve BF, Dickson DW, Halliday G, Taylor J-P, Weintraub D et al (2017) Diagnosis and management of dementia with Lewy bodies: Fourth consensus report of the DLB Consortium. Neurology 89:88-100

61. McKhann GM, Knopman DS, Chertkow H, Hyman BT, Jack CR, Kawas CH et al (2011) The diagnosis of dementia due to Alzheimer's disease: recommendations from the National Institute on Aging-Alzheimer's Association workgroups on diagnostic guidelines for Alzheimer's disease. Alzheimers Dement. https:// doi.org/10.1016/j.jalz.2011.03.005

62. Mesulam MM (1986) Frontal cortex and behavior. Ann Neurol 19:320-325

63. Mesulam MM (1998) From sensation to cognition. Brain 121:1013-1052

64. Mesulam MM, Rogalski EJ, Wieneke C, Hurley RS, Geula C, Bigio EH et al (2014) Primary progressive aphasia and the evolving neurology of the language network. Nat Rev Neurol 10:554-569

65. Mesulam MM, Weintraub S, Rogalski EJ, Wieneke C, Geula C, Bigio EH (2014) Asymmetry and heterogeneity of Alzheimer's and frontotemporal pathology in primary progressive aphasia. Brain 137:1176-1192

66. Montine TJ, Phelps CH, Beach TG, Bigio EH, Cairns NJ, Dickson DW et al (2011) National Institute on Aging-Alzheimer's Association guidelines for the neuropathologic assessment of Alzheimer's disease: a practical approach. Acta Neuropathol 123:1-11

67. Murley AG, Coyle-Gilchrist I, Rouse MA, Jones PS, Li W, Wiggins J et al (2020) Redefining the multidimensional clinical phenotypes of frontotemporal lobar degeneration syndromes. Brain 143:1555-1571

68. Nana AL, Sidhu M, Gaus SE, Hwang J-HL, Li L, Park Y et al (2019) Neurons selectively targeted in frontotemporal dementia reveal early stage TDP-43 pathobiology. Acta Neuropathol 137:27-46

69. Neumann M, Kwong LK, Lee EB, Kremmer E, Flatley A, Xu Y et al (2009) Phosphorylation of S409/410 of TDP-43 is a consistent feature in all sporadic and familial forms of TDP-43 proteinopathies. Acta Neuropathol 117:137-149

70. Neumann M, Lee EB, Mackenzie IR (2021) Frontotemporal dementias, emerging milestones of the 21st century. Adv Exp Med Biol 1281:201-217

71. Nishihira Y, Gefen T, Mao Q, Appin C, Kohler M, Walker J et al (2019) Revisiting the utility of TDP-43 immunoreactive (TDP-43-ir) pathology to classify FTLD-TDP subtypes. Acta Neuropathol 138:167-169

72. Ohm DT, Peterson C, Lobrovich R, Cousins KAQ, Gibbons GS, McMillan CT et al (2020) Degeneration of the locus coeruleus is a common feature of tauopathies and distinct from TDP-43 proteinopathies in the frontotemporal lobar degeneration spectrum. Acta Neuropathol 140:675-693

73. Osher JE, Wicklund AH, Rademaker A, Johnson N, Weintraub S (2008) The mini-mental state examination in behavioral variant frontotemporal dementia and primary progressive aphasia. Am J Alzheimers Dis Other Demen 22:468-473

74. Pasquini L, Nana AL, Toller G, Brown JA, Deng J, Staffaroni A et al (2020) Salience network atrophy links neuron type-specific pathobiology to loss of empathy in frontotemporal dementia. Cereb Cortex 30:5387-5399

75. Pearson RC, Esiri MM, Hiorns RW, Wilcock GK, Powell TP (1985) Anatomical correlates of the distribution of the pathological changes in the neocortex in Alzheimer disease. Proc Natl Acad Sci 82:4531-4534

76. Pievani M, Haan Wd, Wu T, Seeley WW, Frisoni GB (2011) Functional network disruption in the degenerative dementias. Lancet Neurol 10:829-843

77. Pievani M, Filippini N, Heuvel MPVD, Cappa SF, Frisoni GB (2014) Brain connectivity in neurodegenerative diseases-from phenotype to proteinopathy. Nat Rev Neurol 10:620-633

78. Porta S, Xu Y, Restrepo CR, Kwong LK, Zhang B, Brown HJ et al (2018) Patient-derived frontotemporal lobar degeneration brain extracts induce formation and spreading of TDP-43 pathology in vivo. Nat Commun 9:4220 
79. Prudlo J, König J, Schuster C, Kasper E, Büttner A, Teipel S et al (2016) TDP-43 pathology and cognition in ALS. Neurology 87:1019-1023

80. Rabinovici GD, Seeley WW, Kim EJ, Gorno-Tempini ML, Rascovsky K, Pagliaro TA et al (2007) Distinct MRI atrophy patterns in autopsy-proven alzheimer's disease and frontotemporal lobar degeneration. Am J Alzheimers Dis Other Demen 22:474-488

81. Raj A, Kuceyeski A, Weiner M (2012) A network diffusion model of disease progression in dementia. Neuron 73:1204-1215

82. Rascovsky K, Hodges JR, Knopman D, Mendez MF, Kramer JH, Neuhaus J et al (2011) Sensitivity of revised diagnostic criteria for the behavioural variant of frontotemporal dementia. Brain 134:2456-2477

83. Robinson JL, Lee EB, Xie SX, Rennert L, Suh E, Bredenberg C et al (2018) Neurodegenerative disease concomitant proteinopathies are prevalent, age-related and APOE4-associated. Brain 141:2181-2193

84. Rockland KS (2019) What do we know about laminar connectivity? Neuroimage 197:772-784

85. Rogalski E, Rogalski E, Cobia D, Cobia D, Harrison TM, Harrison TM et al (2011) Anatomy of language impairments in primary progressive aphasia. J Neurosci 31:3344-3350

86. Rogalski E, Cobia D, Harrison TM, Wieneke C, Weintraub S, Mesulam MM (2011) Progression of language decline and cortical atrophy in subtypes of primary progressive aphasia. Neurology $76: 1804-1810$

87. Seeley WW (2008) Selective functional, regional, and neuronal vulnerability in frontotemporal dementia. Curr Opin Neurol 21:701-707

88. Seeley WW (2017) Mapping Neurodegenerative Disease Onset and Progression. Cold Spring Harb Perspect Biol 9:a023622

89. Seeley WW (2019) The salience network: a neural system for perceiving and responding to homeostatic demands. J Neurosci 39:9878-9882

90. Seeley WW, Carlin DA, Allman JM, Macedo MN, Bush C, Miller BL et al (2006) Early frontotemporal dementia targets neurons unique to apes and humans. Ann Neurol 60:660-667

91. Seeley WW, Menon V, Schatzberg AF, Keller J, Glover GH, Kenna $\mathrm{H}$ et al (2007) Dissociable intrinsic connectivity networks for salience processing and executive control. J Neurosci 27:2349-2356

92. Seeley WW, Crawford RK, Zhou J, Miller BL, Greicius MD (2009) Neurodegenerative diseases target large-scale human brain networks. Neuron 62:42-52
93. Shipp S (2007) Structure and function of the cerebral cortex. Curr Biol 17:R443-R449

94. Spinelli EG, Mandelli ML, Miller ZA, Santos-Santos MA, Wilson SM, Agosta F et al (2017) Typical and atypical pathology in primary progressive aphasia variants. Ann Neurol 81:430-443

95. Toledo JB, Deerlin VMV, Lee EB, Suh E, Baek Y, Robinson JL et al (2013) A platform for discovery: the university of pennsylvania integrated neurodegenerative disease biobank. Alzheimers Dement 10:477-484.e471

96. Trampel R, Bazin P-L, Pine K, Weiskopf N (2019) In-vivo magnetic resonance imaging (MRI) of laminae in the human cortex. Neuroimage 197:707-715

97. Verny M, Duyckaerts C, Delaère P, He Y, Hauw JJ (1994) Cortical tangles in progressive supranuclear palsy. J Neural Transm Suppl 42:179-188

98. Verny M, Duyckaerts C, Agid Y, Hauw JJ (1996) The significance of cortical pathology in progressive supranuclear palsyClinico-pathological data in 10 cases. Brain 119:1123-1136

99. Wagstyl K, Ronan L, Goodyer IM, Fletcher PC (2015) Cortical thickness gradients in structural hierarchies. Neuroimage 111:241-250

100. Wagstyl K, Lepage C, Bludau S, Zilles K, Fletcher PC, Amunts $\mathrm{K}$ et al (2018) Mapping cortical laminar structure in the 3D BigBrain. Cereb Cortex 28:2551-2562

101. Warren JD, Rohrer JD, Schott JM, Fox NC, Hardy J, Rossor MN (2013) Molecular nexopathies: a new paradigm of neurodegenerative disease. Trends Neurosci 36:561-569

102. Wood EM, Falcone D, Suh E, Irwin DJ, Chen-Plotkin AS, Lee EB et al (2013) Development and validation of pedigree classification criteria for frontotemporal lobar degeneration. JAMA Neuro 70:1411-1417

103. Xie SX, Baek Y, Grossman M, Arnold SE, Karlawish J, Siderowf A et al (2011) Building an integrated neurodegenerative disease database at an academic health center. Alzheimers Dement 7:e84-e93

104. Zhou J, Gennatas Efstathios D, Kramer Joel H, Miller Bruce L, Seeley William W (2012) Predicting Regional Neurodegeneration from the Healthy Brain Functional Connectome. Neuron $73: 1216-1227$

Publisher's Note Springer Nature remains neutral with regard to jurisdictional claims in published maps and institutional affiliations.

\section{Authors and Affiliations}

\section{Daniel T. Ohm ${ }^{1,2}$. Katheryn A. Q. Cousins ${ }^{2} \cdot$ Sharon X. Xie ${ }^{3} \cdot$ Claire Peterson $^{1,2} \cdot$ Corey T. McMillan $^{2}$. Lauren Massimo ${ }^{2} \cdot$ Katya Raskovsky $^{2}$. David A. Wolk ${ }^{4,5}$. Vivianna M. Van Deerlin ${ }^{6}$. Lauren Elman ${ }^{7}$ Meredith Spindler $^{8} \cdot$ Andres Deik $^{8} \cdot$ John Q. Trojanowski ${ }^{4,6,9} \cdot$ Edward B. Lee $^{4,6,9} \cdot$ Murray Grossman $^{2}$. David J. Irwin ${ }^{1,2}$ (1)}

1 Penn Digital Neuropathology Lab, Department of Neurology, Hospital of the University of Pennsylvania, University of Pennsylvania Perelman School of Medicine, 3600 Spruce Street, Philadelphia, PA 19104, USA

2 Penn Frontotemporal Degeneration Center, Department of Neurology, Perelman School of Medicine, University of Pennsylvania, Philadelphia, PA 19104, USA

3 Department of Biostatistics, Epidemiology, and Informatics, Perelman School of Medicine, University of Pennsylvania, Philadelphia, PA 19104, USA
4 Penn Alzheimer's Disease Research Center, Department of Neurology, Perelman School of Medicine, University of Pennsylvania, Philadelphia, PA 19104, USA

5 Penn Memory Center, Department of Neurology, Perelman School of Medicine, University of Pennsylvania, Philadelphia, PA 19104, USA

6 Department of Pathology and Laboratory Medicine, Perelman School of Medicine, University of Pennsylvania, Philadelphia, PA 19104, USA 
7 Comprehensive Amyotrophic Lateral Sclerosis Center,Department of Neurology, Perelman School of Medicine, University of Pennsylvania, Philadelphia, PA 19104, USA

8 Parkinson's Disease and Movement Disorders Center, Department of Neurology, Perelman School of Medicine, University of Pennsylvania, Philadelphia, PA 19107, USA
9 Center for Neurodegenerative Disease Research, Department of Pathology and Laboratory Medicine, Perelman School of Medicine, University of Pennsylvania, Philadelphia, PA 19104, USA 